\title{
Changes in North Atlantic Deep Water strength and bottom water masses during Marine Isotope Stage 3 (45-35 ka BP)
}

\author{
Marcus Gutjahr a,b,*, Babette A.A. Hoogakker ${ }^{c}$, Martin Frank ${ }^{\mathrm{d}}$, I. Nicholas McCave \\ ${ }^{a}$ ETH Zürich, Institute for Isotope Geology and Mineral Resources, 8092 Zürich, Switzerland \\ ${ }^{\mathrm{b}}$ Bristol Isotope Group, Department of Earth Sciences, Wills Memorial Building, Queens Road, Bristol BS8 1RJ, UK \\ ${ }^{\mathrm{c}}$ Godwin Laboratory for Palaeoclimate Research, Department of Earth Sciences, University of Cambridge, Downing Street, Cambridge CB2 3EQ UK \\ ${ }^{\mathrm{d}}$ IFM-GEOMAR, Leibniz Institute of Marine Sciences at the University of Kiel, 24148 Kiel, Germany
}

\section{A R T I C L E I N F O}

\section{Article history:}

Received 1 October 2009

Received in revised form

27 January 2010

Accepted 16 February 2010

\begin{abstract}
A B S T R A C T
The strength of the North Atlantic Meridional Overturning Circulation during climatically highly variable Marine Isotope Stage (MIS) 3 has attracted much attention in recent years. Here we present highresolution $\mathrm{Nd}$ isotope compositions of past seawater derived from authigenic $\mathrm{Fe}-\mathrm{Mn}$ oxyhydroxides recovered from drift sediments on the Blake Ridge in the deep western North Atlantic (ODP Leg 172, Site $1060,3481 \mathrm{~m}$ water depth). The data cover the period from 45 to $35 \mathrm{ka} \mathrm{BP}$, tracing circulation changes during major Heinrich iceberg discharge event 4 (H4, 40-39 ka BP). The Nd isotope record suggests that there was no northern-source water (NSW) mass like modern NADW at the deeper part of Blake Ridge at any time between 45 and $35 \mathrm{ka}$. This is fundamentally different from the hydrographic situation during the Holocene where NADW extends below $4500 \mathrm{~m}$ at this location. The $\varepsilon_{\mathrm{Nd}}$ of past deep water recorded in the Blake Ridge sediments was least radiogenic during Dansgaard/Oeschger (D/O) Interstadial (IS) $8\left(\varepsilon_{\mathrm{Nd}}=-11.3\right)$ and most radiogenic immediately preceding IS $9\left(\varepsilon_{\mathrm{Nd}}=-9.8\right)$. More radiogenic compositions were also recorded during $\mathrm{H} 4\left(-10.2 \leq \varepsilon_{\mathrm{Nd}} \leq-9.9\right)$. The Nd isotope variability in MIS 3 matches that of a physical bottom current strength reconstruction from the same location. Neither record follows the pattern of Northern Hemisphere D/O climatic cycles. In our record, reduced mixing with northern source waters started in stadial 12 and lasted until after $\mathrm{H} 4$ in stadial 9, followed by a rapid increase in NSW contribution thereafter. This major change in the Nd isotope record predates the iceberg discharge event Heinrich 4 by more than $3 \mathrm{ka}$ indicating a shallowing of the water mass boundary between Glacial North Atlantic Intermediate Water and Southern Source Water beneath. This early change in bottom water properties at the deep Blake Ridge suggests that North Atlantic deep water advection may already have decreased several thousand years before the actual iceberg discharge event and associated freshening of the surface waters in the North Atlantic. The change can thus not be attributed to climatic events in the North Atlantic but may be related to changes in flux of deep water from the South.
\end{abstract}

(c) 2010 Elsevier Ltd. All rights reserved.

\section{Introduction}

\subsection{Climate and hydrography}

Marine Isotope Stage 3 (MIS 3) ( $\sim 59$ to $\sim 27 \mathrm{ka} \mathrm{BP}$ ) was a period of extreme climate variability, in particular in the northern hemisphere, as evidenced by both Greenland ice core records (Johnsen et al., 1992; Dansgaard, 1993; NGRIP-Members, 2004) and North Atlantic sea surface temperature reconstructions (Bond et al., 1993;

\footnotetext{
* Corresponding author. Bristol Isotope Group, Department of Earth Sciences, Wills Memorial Building, Queens Road, Bristol BS8 1RJ, UK. Tel.: +44 1179545235.

E-mail address: marcus.gutjahr@bristol.ac.uk (M. Gutjahr).
}

Shackleton et al., 2000). While the fully glacial climate stages MIS 4 and 2 were dominated by relatively stable cold conditions, MIS 3 was special, at least in the North Atlantic region, due to its wellknown succession of suborbital stadial-interstadial periods referred to as Dansgaard-Oeschger (D/O) cycles, which were marked by rapid temperature changes.

Marine Isotope Stages 3 and 2 were also marked by a series of discrete periods of extreme iceberg discharge into the open North Atlantic, the Heinrich Events. These occurred at near regular intervals at the end of longer term $\mathrm{D} / \mathrm{O}$ cooling trends, followed by rapid warming, as recorded both in Greenland ice and North Atlantic sea surface temperatures (Bond et al., 1993; Hemming, 2004). The exact cause for these iceberg discharge 
events is not yet resolved but Atlantic water mass stratification and overturning circulation clearly changed during these events (Vidal et al., 1997; Rasmussen et al., 2003; McManus et al., 2004).

Northern hemisphere climate has been closely coupled to the strength of Atlantic Meridional Overturning Circulation (MOC) (Clark et al., 2002). The distribution of northern-sourced versus southern-sourced deep water in the Atlantic varied due to changing fluxes and densities of the different water masses over the last glacial cycle (Adkins et al., 2002; Rahmstorf, 2002). Evidence from a variety of geochemical proxies suggests that northern-source deep water (NSW) advection in the North Atlantic was active but significantly shallower during the LGM compared with the Holocene and the Last Interglacial (Eemian) situation (Keigwin et al., 1994; Oppo and Lehman, 1995; Rasmussen et al., 2003; LynchStieglitz et al., 2007). Holocene North Atlantic Deep Water (NADW) occupies a significantly larger fraction of the deep Atlantic than its glacial counterpart, the Glacial North Atlantic Intermediate Water (GNAIW) (e.g. Curry and Oppo, 2005). The shallow GNAIW advection cell was underlain by a large body of most likely Southern Ocean-derived water (SSW). The boundary between northern- and southern-sourced water in the deep North Atlantic basin during the LGM was probably situated between 2000 and $2500 \mathrm{~m}$ water depth and possibly as deep as $2800 \mathrm{~m}$ in the Northeast Atlantic (Lynch-Stieglitz et al., 2007; Yu et al., 2008), whereas today NADW occupies almost the entire water column in the western North Atlantic (Amos et al., 1971; Schmitz and McCartney, 1993).

Compared with the LGM benthic foraminiferal carbon isotope records suggest that during MIS 3 NSW occupied a larger proportion of the North Atlantic at least during Greenland Interstadials (Oppo and Lehman, 1995; Vidal et al., 1997; Elliot et al., 2002; Hagen and Keigwin, 2002; Rasmussen et al., 2003). At the Blake Ridge in the western North Atlantic, Hagen and Keigwin (2002) used benthic foraminiferal carbon isotope evidence to infer the presence of NADW during interstadials down to $\sim 3000 \mathrm{~m}$ water depth and its replacement by SSW during stadials.

Constraining the state of the Atlantic MOC during Heinrich iceberg discharge events is more difficult. While it appears that Atlantic MOC generally weakened during Heinrich Events (e.g., Oppo and Lehman, 1995; Vidal et al., 1997), the magnitude and timing of these MOC reductions is not resolved. McManus et al. (2004) suggested a near total collapse of Atlantic MOC during Heinrich event 1 based on a sedimentary Pa/Th record from the Bermuda Rise. However, since the adsorbed sedimentary Pa/Th signal is also highly sensitive to particle compositions (biogenic silica content) such an interpretation has to be treated with caution and Atlantic MOC reduction may not have been so severe (Chase et al., 2002; Hall et al., 2006; Keigwin and Boyle, 2008; Gherardi et al., 2009; Lippold et al., 2009). In addition, several of the major Heinrich IRD discharge events (H1, H2, H4) were apparently accompanied by meltwater precursor events originating from the Northwest European Ice Sheet (NWEIS) suggesting that ice sheet collapse of European origin may have pre-conditioned the MOC in the North Atlantic (Peck et al., 2007b).

While change in the MOC is clearly evidenced, chronological constraints for some MIS 3 sediment cores are, however, not satisfactory, or sampling and time resolution was not high enough to determine exact sequence of events among climate, deep water mass distribution, high iceberg discharge and the vigour of bottom water flow at sub-millennial resolution. It is of broad interest to better understand the origin and residence time of deep glacial Atlantic water masses in view of their potential to store the respired carbon that was drawn from the atmosphere during the last glacial cycle (e.g. Brovkin et al., 2007).

\subsection{Water-mass tagging by $\mathrm{Nd}$ isotopes}

The aim of our study is to evaluate provenance and mixing proportions of water masses in the deep western North Atlantic during MIS 3 using the authigenic Fe-Mn oxyhydroxide-derived $\mathrm{Nd}$ isotope composition of past deepwater recorded in marine sediments (Palmer and Elderfield, 1985; Rutberg et al., 2000; Bayon et al., 2002; Piotrowski et al., 2005; Gutjahr et al., 2007, 2008; Klevenz et al., 2008; Roberts et al., 2010). The isotopic composition of $\mathrm{Nd}$ in seawater is primarily set by riverine continental runoff (Frank, 2002; Goldstein and Hemming, 2003), exchange with shelf sediments at the ocean's boundaries (Lacan and Jeandel, 2005a), dust input and possibly submarine groundwater discharge (Johannesson and Burdige, 2007). The Nd isotopic signature of a water mass is controlled by the average age and $\mathrm{Sm} / \mathrm{Nd}$ of the continental input sources since ${ }^{143} \mathrm{Nd} /{ }^{144} \mathrm{Nd}$ is a function of the decay of ${ }^{147} \mathrm{Sm}$ to ${ }^{143} \mathrm{Nd}\left(\mathrm{T}_{1 / 2}=106 \mathrm{Ga}\right)$. The $\mathrm{Nd}$ isotope composition is given in epsilon notation by normalisation to the Chondrite Uniform Reservoir (CHUR):

$\varepsilon_{\mathrm{Nd}}=\left[\frac{{ }^{143} \mathrm{Nd} /{ }^{144} \mathrm{Nd}_{\text {sample }}}{{ }^{143} \mathrm{Nd} /{ }^{144} \mathrm{Nd}_{\mathrm{CHUR}}}-1\right] \times 10^{4}$

with ${ }^{143} \mathrm{Nd} /{ }^{144} \mathrm{Nd}_{\mathrm{CHUR}}=0.512638$ (Jacobsen and Wasserburg, 1980). Since continental crust has low $\mathrm{Sm} / \mathrm{Nd}$, old continental provinces such as those bordering the Labrador Sea have very unradiogenic Nd isotopic compositions leading to very negative $\varepsilon_{\mathrm{Nd}}$. Conversely, young mantle-derived rocks such as volcanic island arcs display very radiogenic $\mathrm{Nd}$ isotope compositions. Neodymium in the open ocean behaves quasi-conservatively with a residence time of 500-2000 years (Jeandel et al., 1995; Tachikawa et al., 2003).

The Nd isotopic composition of NADW is initially set through the mixture of Labrador Sea Water with waters from the Nordic Seas giving it a characteristic unradiogenic Nd isotope composition, by which it can be traced along its export pathway towards the Southern Ocean. Although NADW consists of water masses of variable origin (e.g. Labrador Sea Water, Island-Scotland Overflow Water, Denmark Strait Overflow Water) its Nd isotope composition is rather uniform with a vertical variability of $1.3 \varepsilon_{\mathrm{Nd}}$ $\left(-13.2 \geq \varepsilon_{\mathrm{Nd}} \geq-14.5\right)$ in the vicinity of Orphan Knoll (Lacan and Jeandel, 2005b). In the subtropical NW Atlantic close to the Blake Ridge it is fully homogenised, having an $\varepsilon_{\mathrm{Nd}}$ of $-13.5 \pm 0.5$ (Piepgras and Wasserburg, 1987), which is mainly achieved though effective recirculation in gyres in the subpolar and western North Atlantic (e.g. Smethie et al., 2000). Other water masses in the North Atlantic (Lacan and Jeandel, 2004a), the Nordic Seas Overflow Water in the North Atlantic (Lacan and Jeandel, 2004b) or Mediterranean Outflow Water (Tachikawa et al., 2004) have more radiogenic $\mathrm{Nd}$ isotopic compositions $\left(-7.7 \geq \varepsilon_{\mathrm{Nd}} \geq-10.7\right)$, unless they have been modified by mixing with NADW in the subpolar North Atlantic (Lacan and Jeandel, 2004a). At present, Antarctic Intermediate Water (AAIW) and Antarctic Bottom Water (AABW) formed in the Southern Ocean have Nd isotopic compositions in the range of $-7 \geq \varepsilon_{\mathrm{Nd}} \geq-11$ at their source areas (Jeandel, 1993; Rickli et al., 2009).

Along continental margins, particle-seawater interaction was found to be an important process capable of modifying the dissolved deep water $\mathrm{Nd}$ isotopic composition and was termed boundary exchange (Lacan and Jeandel, 2005a) or reversible scavenging (Siddall et al., 2008). Its importance in altering the ambient deep water $\varepsilon_{\mathrm{Nd}}$ could potentially be crucial and has to be evaluated for core locations underneath oceanic deep boundary currents, such as the ones investigated here. The bottom water $\varepsilon_{\mathrm{Nd}}$ is incorporated into sedimentary authigenic Fe-Mn oxyhydroxides after 
deposition, and in deep open ocean settings this $\mathrm{Nd}$ isotopic signature can be reliably extracted (e.g. Bayon et al., 2002; Piotrowski et al., 2005; Gutjahr et al., 2007). A major deglacial shift of $3-4 \varepsilon_{\mathrm{Nd}}$ units towards more radiogenic signatures of bottom waters in deep NE and NW Atlantic core sites was interpreted as increased presence of Southern Source Water during the LGM and the deglaciation (Gutjahr et al., 2008; Yu et al., 2008; Roberts et al., 2010). In the deep NE Atlantic, radiogenic $\varepsilon_{\mathrm{Nd}}$ signatures as high as -8.4 during the LGM changed towards less radiogenic values as low as -11.2 during the early Holocene (Yu et al., 2008). The deeper Blake Ridge in the subtropical NW Atlantic recorded a change from radiogenic $\varepsilon_{\mathrm{Nd}}$ of about -10.3 during the LGM to unradiogenic $\varepsilon_{\mathrm{Nd}}$ as low as -14.0 during the early Holocene tracing the establishment of deep NADW (Gutjahr et al., 2008). Roberts et al. (2010) recently reported initial deglacial deep water NADW ventilation at the Bermuda Rise as early as the Bølling-Allerød interstadial ( 14.5-12.9 ka BP).

The present study focuses on the variability of past seawater $\mathrm{Nd}$ isotope compositions recorded at the deeper Blake Ridge at ODP Site 1060 (3481 m) (Fig. 1) surrounding Heinrich Event 4 at submillennial resolution. These data are compared with a sortable silt mean size $(\overline{\mathrm{SS}})$ record from the same site (Hoogakker et al., 2007) and epibenthic carbon isotope data from nearby core sites along the Blake Ridge (Hagen and Keigwin, 2002) in order to better constrain the deep water mass distribution in the western North Atlantic during this climatically highly variable period.

\section{Material and methods}

Marine sediment samples used here were cored during ODP Leg 172 at Site 1060 ( $30^{\circ} 46^{\prime} \mathrm{N}, 74^{\circ} 28^{\prime} \mathrm{W}, 3481 \mathrm{~m}$ water depth) on Blake Ridge in the western North Atlantic (Fig. 1). In total 22 individual samples with a depositional age between $45 \mathrm{ka} \mathrm{BP}$ and $35 \mathrm{ka} \mathrm{BP}$ were analysed to obtain the Nd isotope composition of past deep water from authigenic Fe-Mn oxyhydroxides. Additionally the extracted major and trace element concentrations were measured and the leached $\mathrm{Sr}$ isotope compositions were determined for the evaluation of the seawater origin of the extracted signal following the extraction scheme described in Gutjahr et al. (2007) with minor modifications, as briefly outlined below.

Carbonate was removed using a Na acetate buffer in a shaker, followed by the removal of loosely adsorbed metals employing a $1 \mathrm{M} \mathrm{MgCl}_{2}$ solution in a shaker for one hour. Following centrifugation and triple rinses in deionised water (Milli-Q system), the $\mathrm{Fe}-\mathrm{Mn}$ oxyhydroxides were dissolved by leaching the samples for one hour in a shaker in a $0.05 \mathrm{M}$ hydroxylamine hydrochloride $(\mathrm{HH})$ - 15\% distilled acetic acid. In contrast to the method described in Gutjahr et al. (2007), neither Na-EDTA nor $\mathrm{NaOH}$ were added to the reductive leaching solution and the samples were only leached for one hour as opposed to three hours, which led to significantly reduced $\mathrm{Nd}$ yields compared with this earlier study. Na-EDTA was not added to the reductive leaching solution since only $\mathrm{Nd}$ and $\mathrm{Sr}$ were extracted for purification and isotopic analyses, and partial readsorption of leached REEs is not a major issue compared with other more particle-reactive elements such as Th. The extracted leached sample solutions were almost completely used for $\mathrm{Nd}$ and $\mathrm{Sr}$ isotopic measurements but a small fraction was kept for major and trace element concentration measurements.

Strontium and Neodymium were extracted from the $\mathrm{Fe}-\mathrm{Mn}$ oxyhydroxide matrix following the standard prodecures of Horwitz et al. (1992) for Sr and those of Cohen et al. (1988) for Nd. Total analytical blanks were on the order of $\sim 32 \mathrm{pg}$ for $\mathrm{Sr}$ and below $10 \mathrm{pg}$ for $\mathrm{Nd}$ and are negligible.

Measurements of the $\mathrm{Sr}$ and $\mathrm{Nd}$ isotopic compositions were carried out on a Nu Plasma MC-ICPMS at ETH Zürich using the exponential law to correct for instrumental mass fractionation. Interference-corrected ${ }^{87} \mathrm{Sr} /{ }^{86} \mathrm{Sr}$ was adjusted for instrumentinduced mass bias by normalising to ${ }^{86} \mathrm{Sr} /{ }^{88} \mathrm{Sr}$ of 0.1194 . All reported $\mathrm{Sr}$ isotope results were normalised to ${ }^{87} \mathrm{Sr} /{ }^{86} \mathrm{Sr}=0.710245$ for the NIST SRM987 $\mathrm{Sr}$ standard. The long-term reproducibility for repeated measurements of the NIST SRM987 $\mathrm{Sr}$ standard was $0.000025(2 \sigma ; n=70)$. Measured ${ }^{143} \mathrm{Nd} /{ }^{144} \mathrm{Nd}$ was corrected for the instrumental mass bias applying a ${ }^{146} \mathrm{Nd} /{ }^{144} \mathrm{Nd}$ of 0.7219 . All

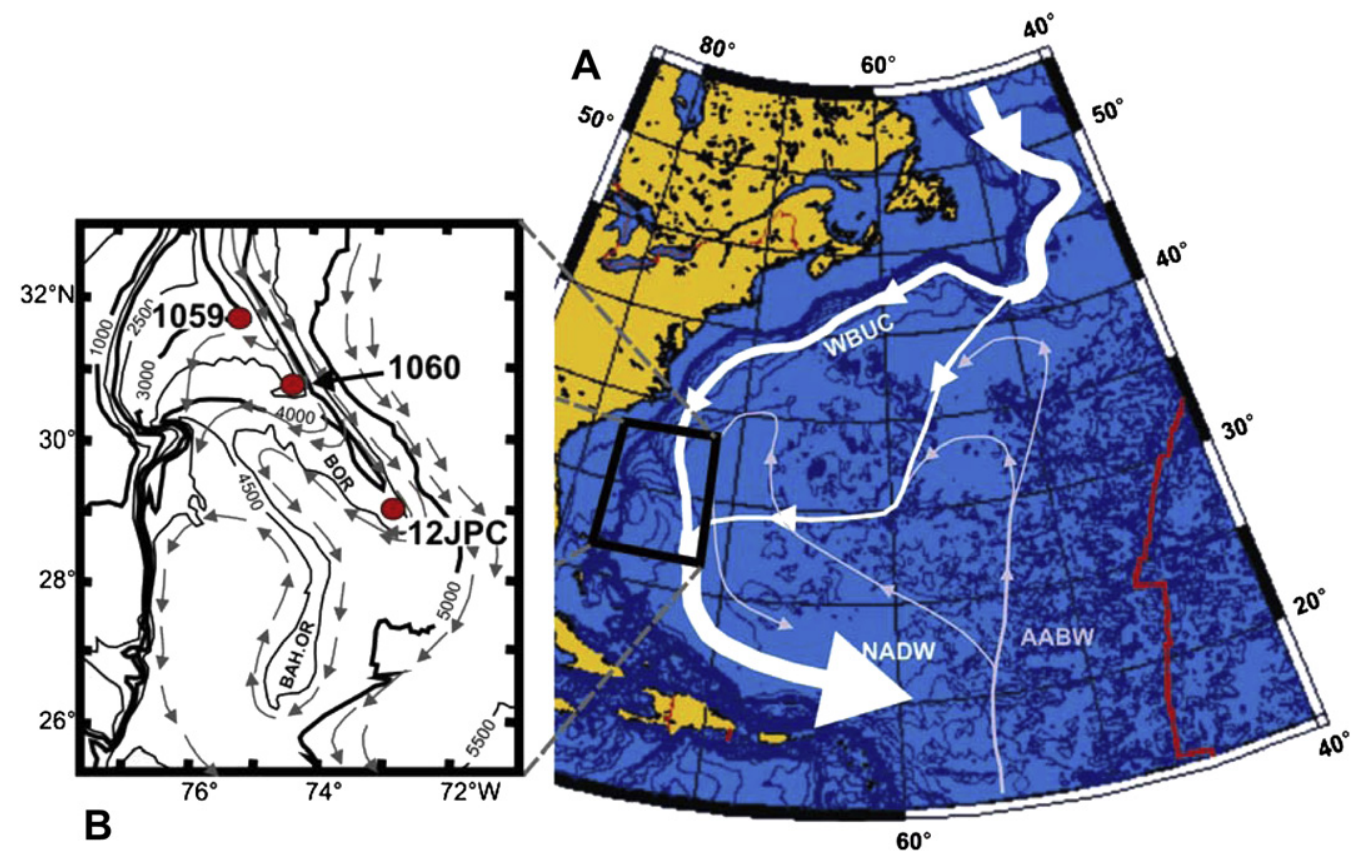

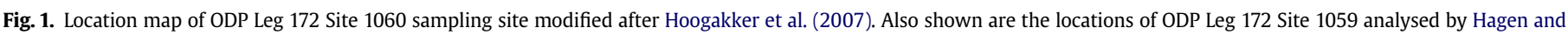
Keigwin (2002) as well as Site KNR140/12JPC (Gutjahr et al., 2008). 
reported $\mathrm{Nd}$ isotope results were normalised to a ${ }^{143} \mathrm{Nd} /{ }^{144} \mathrm{Nd}$ of 0.512115 for the JNdi- 1 standard (Tanaka et al., 2000). The long-term reproducibility for repeated measurements of a $50 \mathrm{ppb}$ JNdi-1 standard solution was $\pm 0.27 \varepsilon_{\mathrm{Nd}}(2 \sigma ; \mathrm{n}=70)$. The $\mathrm{Sr}$ and $\mathrm{Nd}$ isotopic results are shown in Table 1 . For the evaluation of the extraction procedure five duplicates have been processed through chemistry separately and are shown alongside the remaining data in Tables 1 and 2 and Fig. 5. The Nd isotopic compositions of USGS standard nodules NOD-P-1 and NOD-A-1 measured during this study reproduced at ${ }^{143} \mathrm{Nd} /{ }^{144} \mathrm{Nd}$ of $0.512439 \pm 8(\mathrm{NOD}-\mathrm{P}-1)$ and $0.512142 \pm 6$ (NOD-A-1) and hence within $14 \mathrm{ppm}$ and $12 \mathrm{ppm}$ to previously reported Nd isotope compositions (Foster and Vance, 2006).

Major and trace element concentrations were measured on all $\mathrm{Fe}-\mathrm{Mn}$ oxyhydroxide leachates using a Vista axially viewed plasma (AX) charge coupled device (CCD) simultaneous ICP-AES and a PerkinElmer SCIEX Elan DRC II quadrupole ICP-MS at the Department of Earth Sciences, University of Cambridge (UK). Longterm analytical precision of the ICP-AES is $\sim 5 \%$. Results for USGS rock standards AGV-2 and BCR-2, as determined by ICPMS at the University of Cambridge, are presented in Table 2. ICP-MS external reproducibility, based on replicate analysis of $\mathrm{BCR}-2$ and repeat samples within batches, varies between 0.5 and $5 \%$ for most analytes. Compositions only deviate from published values by $1-11 \%$ and are thus in good agreement. Cross-calibration between the ICP-OES and ICP-MS data was done by comparison of the respective concentrations obtained for selected elements measured on both instruments. Respective concentrations vary within $0.10-4.53 \%$ for $\mathrm{Mn}$, within $0.03-5.96 \%$ for $\mathrm{Sr}$, and within $0.38-5.78 \%$ for $\mathrm{Ba}(\mathrm{n}=27)$.

In addition, USGS ferromanganese nodule standards NOD-A-1 and NOD-P-1 were subjected to the same leaching procedure that was applied to the Blake Ridge sediments. The leaching results are shown in Tables 1, 2 and Fig. 2. The release of rare earth elements
(REEs) occurred essentially congruently since the Post Archean Average Australian Shale (PAAS)-normalised REE patterns agree well with the respective recommended relative concentrations given in Axelsson et al. (2002) (Fig. 2a), albeit having significantly reduced total concentrations (Fig. 2b). Only approximately $1.2-1.6 \%$ of the ferromanganese nodule material was extracted during the one-hour reductive leaching procedure (Fig. 2 b).

The chronologies for core 1060 follows Hoogakker et al. (2007) who tie in the initial age model for Site 1060 presented by Vautravers et al. (2004), based on correlation between the percentage of warm surface-dwelling planktonic foraminifera, to the foraminiferal oxygen isotope $\left(\delta^{18} \mathrm{O}\right)$ record of core MD95-2042 off Portugal, with the revised age scale for the Greenland ice cores (SFCP04 timescale) (Shackleton et al., 2004). The SFCP04 timescales is based on ${ }^{14} \mathrm{C}$ dating of the planktonic foraminiferal species Globigerina bulloides, calibrated by pairs of ${ }^{14} \mathrm{C}$ and ${ }^{230} \mathrm{Th}$ measurements on corals (Shackleton et al., 2004). Benthic foraminiferal $\delta^{18} \mathrm{O}$ records from ODP Site 1060 (west Atlantic) and MD95-2042 (east Atlantic) show very similar variations between 35 and $45 \mathrm{kyr}$, confirming the age model is robust (Hoogakker et al., 2007).

\section{Results}

\subsection{Extracted leachate major and trace element composition}

The major element concentrations displayed in Table 2 suggest that the major extracted phases are Fe oxyhydroxides since the Fe/ Mn ratio for all leached samples averages $44.0 \pm 11.7$ (2 s.d.), which is very different from the respective $\mathrm{Fe} / \mathrm{Mn}$ of the two leached ferromanganese nodule standards $(0.45$ and 0.14$)$. The REE patterns of the leached Fe-Mn oxyhydroxides show very clear PAAS-normalised Middle REE enrichment (Fig. 3). The extracted REE concentrations, when normalised to the amount of sediment used

Table 1

Neodymium and strontium isotope results of all analysed Fe-Mn oxyhydroxide samples, USGS ferromanganese nodule standard material NOD-A-1 and NOD-P-1.

\begin{tabular}{|c|c|c|c|c|c|}
\hline IODP number & & SFCP04 age (kaBP) & ${ }^{143} \mathrm{Nd} /{ }^{144} \mathrm{Nd}$ & $\varepsilon_{\mathrm{Nd}}$ & ${ }^{87} \mathrm{Sr} /{ }^{86} \mathrm{Sr}$ \\
\hline \multicolumn{6}{|c|}{ IODP 172 1060A-2H- } \\
\hline $2 \mathrm{~W}$ & $38-40 \mathrm{~cm}$ & 35.04 & $0.512097 \pm 8$ & -10.5 & $0.71285 \pm 1.4$ \\
\hline $2 \mathrm{~W}$ & $78-80 \mathrm{~cm}$ & 36.02 & $0.512075 \pm 9$ & -11.0 & $0.71273 \pm 1.7$ \\
\hline $3 \mathrm{~W}$ & $9-11 \mathrm{~cm}$ & 37.95 & $0.512064 \pm 8$ & -11.2 & $0.71311 \pm 1.2$ \\
\hline $3 W$ & $9-11 \mathrm{~cm}$ duplicate & & $0.512067 \pm 6$ & -11.1 & $0.71322 \pm 1.4$ \\
\hline $3 \mathrm{~W}$ & $18-20 \mathrm{~cm}$ & 38.36 & $0.512058 \pm 8$ & -11.3 & $0.71266 \pm 1.3$ \\
\hline $3 \mathrm{~W}$ & $34-36 \mathrm{~cm}$ & 39.10 & $0.512130 \pm 11$ & -9.9 & $0.71337 \pm 1.3$ \\
\hline $3 \mathrm{~W}$ & $42-44 \mathrm{~cm}$ & 39.22 & $0.512115 \pm 10$ & -10.2 & $0.71383 \pm 1.9$ \\
\hline $3 \mathrm{~W}$ & $50-52 \mathrm{~cm}$ & 39.35 & $0.512130 \pm 6$ & -9.9 & $0.71320 \pm 1.0$ \\
\hline \multirow[t]{2}{*}{$3 \mathrm{~W}$} & $58-60 \mathrm{~cm}$ & 39.47 & $0.512142 \pm 11$ & -9.7 & $0.71373 \pm 1.3$ \\
\hline & $58-60 \mathrm{~cm}$ duplicate & & $0.512115 \pm 9$ & -10.2 & n.a. \\
\hline $3 \mathrm{~W}$ & $66-68 \mathrm{~cm}$ & 39.60 & $0.512114 \pm 8$ & -10.2 & $0.71361 \pm 1.7$ \\
\hline $3 \mathrm{~W}$ & $74-76 \mathrm{~cm}$ & 39.72 & $0.512129 \pm 5$ & -9.9 & $0.71387 \pm 1.7$ \\
\hline $3 W$ & 74-76 duplicate & & $0.512129 \pm 7$ & -9.9 & $0.71317 \pm 1.3$ \\
\hline $3 \mathrm{~W}$ & $100-102 \mathrm{~cm}$ & 40.10 & $0.512115 \pm 4$ & -10.2 & $0.71413 \pm 1.5$ \\
\hline $3 \mathrm{~W}$ & $114-116 \mathrm{~cm}$ & 40.35 & $0.512107 \pm 9$ & -10.4 & $0.71452 \pm 1.5$ \\
\hline $3 \mathrm{~W}$ & $130-132 \mathrm{~cm}$ & 40.60 & $0.512118 \pm 12$ & -10.2 & $0.71290 \pm 1.4$ \\
\hline $3 \mathrm{~W}$ & $138-140 \mathrm{~cm}$ & 40.72 & $0.512136 \pm 6$ & -9.8 & $0.71363 \pm 1.7$ \\
\hline $4 \mathrm{~W}$ & $2-4 \mathrm{~cm}$ & 41.20 & $0.512109 \pm 14$ & -10.3 & $0.71276 \pm 1.3$ \\
\hline $4 W$ & $2-4 \mathrm{~cm}$ duplicate & & $0.512101 \pm 10$ & -10.5 & $0.71261 \pm 1.2$ \\
\hline $4 \mathrm{~W}$ & $22-24 \mathrm{~cm}$ & 42.02 & $0.512084 \pm 14$ & -10.8 & $0.71346 \pm 1.2$ \\
\hline $4 W$ & $22-24 \mathrm{~cm}$ duplicate & & $0.512105 \pm 18$ & -10.4 & $0.71363 \pm 1.6$ \\
\hline $4 \mathrm{~W}$ & $42-44 \mathrm{~cm}$ & 42.50 & $0.512097 \pm 8$ & -10.5 & $0.71417 \pm 1.3$ \\
\hline $4 \mathrm{~W}$ & $62-64 \mathrm{~cm}$ & 43.04 & $0.512121 \pm 7$ & -10.1 & $0.71339 \pm 1.2$ \\
\hline $4 \mathrm{~W}$ & $74-76 \mathrm{~cm}$ & 43.49 & $0.512100 \pm 11$ & -10.5 & $0.71379 \pm 1.4$ \\
\hline $4 W$ & $74-76 \mathrm{~cm}$ duplicate & & $0.512104 \pm 16$ & -10.4 & $0.71322 \pm 1.5$ \\
\hline $4 \mathrm{~W}$ & $94-96 \mathrm{~cm}$ & 44.07 & $0.512090 \pm 6$ & -10.7 & $0.71366 \pm 1.4$ \\
\hline $4 \mathrm{~W}$ & $114-116 \mathrm{~cm}$ & 44.47 & $0.512061 \pm 11$ & -11.2 & $0.71288 \pm 1.6$ \\
\hline $4 \mathrm{~W}$ & $142-144 \mathrm{~cm}$ & 45.08 & $0.512067 \pm 7$ & -11.1 & $0.71278 \pm 1.6$ \\
\hline \multicolumn{6}{|c|}{ USGS Ferromanganese nodule standards } \\
\hline NOD-A-1 & (leached) & & $0.512142 \pm 6$ & -9.7 & $0.70915 \pm 1.2$ \\
\hline NOD-P-1 & (leached) & & $0.512439 \pm 8$ & -3.9 & $0.70914 \pm 1.5$ \\
\hline
\end{tabular}


Table 2

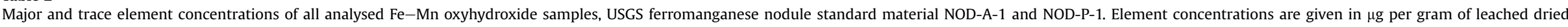
powdered sediment for major elements and in ng per gram for trace elements. Also shown are the USGS trace element concentrations of AGV-2 and BGR-2 analysed as secondary external standard.

\begin{tabular}{|c|c|c|c|c|c|c|c|c|c|c|c|c|c|c|c|c|c|c|c|c|c|c|c|c|c|c|c|c|c|c|}
\hline \multicolumn{2}{|c|}{ ODP sample number } & \multirow{3}{*}{$\begin{array}{l}\begin{array}{l}\mathrm{Al} \\
(\mu \mathrm{g} / \mathrm{g})\end{array} \\
235\end{array}$} & \multirow{3}{*}{$\begin{array}{l}\begin{array}{l}\mathrm{Fe} \\
(\mu \mathrm{g} / \mathrm{g})\end{array} \\
437\end{array}$} & \multirow{2}{*}{$\begin{array}{l}\begin{array}{l}\mathrm{Mn} \\
(\mu \mathrm{g} / \mathrm{g})\end{array} \\
\end{array}$} & \multirow{2}{*}{$\begin{array}{l}\mathrm{Ti} \\
\text { (ng/g) } \\
\end{array}$} & \multirow{2}{*}{$\begin{array}{l}\mathrm{Sr} \\
(\mathrm{ng} / \mathrm{g})\end{array}$} & \multirow{2}{*}{$\begin{array}{l}\mathrm{Y} \\
(\mathrm{ng} / \mathrm{g}) \\
\end{array}$} & \multirow{2}{*}{$\begin{array}{l}\mathrm{La} \\
(\mathrm{ng} / \mathrm{g})\end{array}$} & \multirow[t]{2}{*}{$\begin{array}{l}\mathrm{Ce} \\
\text { (ng/g) }\end{array}$} & \multirow[t]{2}{*}{$\begin{array}{l}\mathrm{Pr} \\
\text { (ng/g) }\end{array}$} & \multirow{2}{*}{$\begin{array}{l}\begin{array}{l}\mathrm{Nd} \\
(\mathrm{ng} / \mathrm{g})\end{array} \\
\end{array}$} & \multirow[t]{2}{*}{$\begin{array}{l}\mathrm{Sm} \\
(\mathrm{ng} / \mathrm{g}) \\
\end{array}$} & \multirow{2}{*}{$\begin{array}{l}\begin{array}{l}\mathrm{Eu} \\
(\mathrm{ng} / \mathrm{g})\end{array} \\
\end{array}$} & \multirow{2}{*}{$\begin{array}{l}\mathrm{Gd} \\
\text { (ng/g) } \\
\end{array}$} & \multirow{2}{*}{$\begin{array}{l}\mathrm{Tb} \\
\text { (ng/g) } \\
\end{array}$} & \multirow{2}{*}{$\begin{array}{l}\text { Dy } \\
\text { (ng/g) }\end{array}$} & \multirow{2}{*}{$\begin{array}{l}\begin{array}{l}\text { Ho } \\
\text { (ng/g) }\end{array} \\
\end{array}$} & $\begin{array}{l}\text { Er } \\
\text { (ng/g) }\end{array}$ & $\begin{array}{l}\mathrm{Tm} \\
(\mathrm{ng} / \mathrm{g})\end{array}$ & $\begin{array}{l}\mathrm{Yb} \\
(\mathrm{ng} / \mathrm{g})\end{array}$ & $\begin{array}{l}\mathrm{Lu} \\
(\mathrm{ng} / \mathrm{g})\end{array}$ & $\begin{array}{l}\mathrm{Tl} \\
\text { (ng/g) }\end{array}$ & $\begin{array}{l}\mathrm{Pb} \\
(\mathrm{ng} / \mathrm{g})\end{array}$ & $\begin{array}{l}\text { Th } \\
\text { (ng/g) }\end{array}$ & $\begin{array}{l}\mathrm{U} \\
(\mathrm{ng} / \mathrm{g})\end{array}$ & $\mathrm{Al} / \mathrm{Nd}$ & $\mathrm{Al} / \mathrm{Pb}$ & Ti/Nd & $\mathrm{Al} / \mathrm{Th}$ & $\mathrm{Fe} / \mathrm{Mn}$ \\
\hline IODP 172 & $1060 \mathrm{~A}-2 \mathrm{H}-$ & & & & & & & & & & & & & & & & & & & & & & & & & & & & & \\
\hline $2 \mathrm{~W}$ & $38-40 \mathrm{~cm}$ & & & 9.7 & 180 & 747 & 672 & 346 & 974 & 145 & 780 & 232 & 44 & 265 & 31 & 144 & 22 & 49 & 6.1 & 31 & 4.2 & 2.9 & 420 & 39 & 25 & 302 & 561 & 0.23 & 6055 & 44.9 \\
\hline $2 \mathrm{~W}$ & $78-80 \mathrm{~cm}$ & 178 & 458 & 13.1 & 212 & 775 & 519 & 299 & 813 & 117 & 627 & 171 & 33 & 189 & 23 & 108 & 17 & 39 & 4.7 & 26 & 2.9 & 5.9 & 468 & 26 & 25 & 283 & 379 & 0.34 & 6775 & 35.1 \\
\hline $3 \mathrm{~W}$ & $9-11 \mathrm{~cm}$ & & 413 & 10.4 & 325 & 1011 & 529 & 295 & 818 & 117 & 620 & 168 & 32 & 0 & 23 & 103 & 17 & 38 & 4.8 & 27 & 3.5 & 2.8 & 344 & 24 & 23 & 313 & 564 & 0.52 & 036 & 39.8 \\
\hline $3 w$ & $\begin{array}{l}9-11 \mathrm{~cm} \\
\text { duplicate }\end{array}$ & 78 & 405 & 10.7 & 208 & 633 & 504 & 297 & 805 & 115 & 576 & 161 & 30 & 176 & 21 & 101 & 16 & 38 & 4.7 & 26 & 3.0 & 2.4 & 340 & 25 & 20 & 309 & 524 & 0.36 & 7153 & 37.9 \\
\hline $3 \mathrm{~W}$ & $18-20 \mathrm{~cm}$ & 179 & 352 & 8.8 & 275 & 1110 & 528 & 297 & 837 & 118 & 635 & 173 & 35 & 197 & 23 & 105 & $1 \varepsilon$ & 38 & 4.8 & 25 & 3.4 & 4.1 & 336 & 22 & 30 & 282 & 532 & 0.43 & 8253 & 39.8 \\
\hline $3 \mathrm{~W}$ & $-36 \mathrm{~cm}$ & & & & 352 & 1147 & 594 & 306 & 912 & 136 & 744 & 221 & 4 & . & 29 & 12 & & 43 & 5.5 & & . & 3.4 & 394 & 31 & 2 & 326 & 614 & 47 & 305 & 52.3 \\
\hline $3 \mathrm{~W}$ & $42-44 \mathrm{~cm}$ & 261 & 48 & 7.1 & 244 & 1145 & 547 & 296 & 881 & 126 & 694 & 211 & 41 & 238 & 27 & 120 & 19 & 41 & 4.9 & 25 & 3.2 & 3.2 & 367 & 32 & 26 & 377 & 712 & 0.35 & 8051 & 48.9 \\
\hline $3 \mathrm{~W}$ & $50-52 \mathrm{~cm}$ & & 37 & 9.4 & 165 & 779 & 625 & 336 & 976 & 141 & 756 & 221 & 41 & 242 & 29 & 131 & & 45 & 5.4 & 36 & 3.9 & 2.8 & 417 & 36 & 2 & 363 & 58 & 22 & 7602 & 41.4 \\
\hline $3 \mathrm{~W}$ & $5 \varepsilon$ & & 39 & 8.1 & 279 & 1138 & 611 & 317 & 963 & 149 & 781 & 236 & 47 & 4 & 31 & 140 & 2 & 45 & 55 & 2 & 3.7 & 3.1 & 435 & 36 & & 354 & 36 & 36 & 02 & 48.7 \\
\hline $3 \mathrm{~V}$ & 6 & & 360 & - & 249 & 1062 & 513 & 259 & 799 & 118 & 3 & 2 & 4 & & 26 & & & 3 & & & & 7 & 421 & 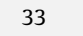 & & & & & & 48.8 \\
\hline $3 \mathrm{~W}$ & $74-76 \mathrm{~cm}$ & 274 & 399 & 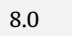 & 306 & 1125 & 601 & 311 & 967 & 136 & 767 & 236 & 45 & 71 & 31 & 137 & 2 & 47 & J.4 & 25 & 3.6 & 3.0 & 452 & 37 & 20 & 357 & 606 & 40 & 7463 & 49.9 \\
\hline $3 W$ & $\begin{array}{l}74-76 \\
\text { duplicate }\end{array}$ & 303 & 477 & 10.5 & 238 & 964 & 657 & 330 & 1038 & 144 & 799 & 243 & 47 & 276 & 31 & 142 & 23 & 45 & 5.9 & 31 & 3.9 & 2.9 & 502 & 39 & 24 & 379 & 603 & 0.30 & 7833 & 45.6 \\
\hline $3 \mathrm{~W}$ & $98-100 \mathrm{~cm}$ & 316 & 59 & 12.9 & 310 & 1152 & 636 & 345 & 1023 & 143 & 754 & 226 & 43 & 25 & 29 & 134 & 21 & 46 & 5. & 29 & 3.5 & 3.5 & 458 & 35 & 26 & 20 & 691 & 41 & 9041 & 35.7 \\
\hline $3 \mathrm{n}$ & & 241 & & 9. & 265 & 933 & 510 & 286 & 849 & 121 & 0 & 182 & 3 & & 2 & 10 & 1 & 3 & 4.4 & 2 & 3.2 & 2.9 & 400 & 32 & & 9 & 3 & 33 & 7490 & 42.9 \\
\hline $3 \mathrm{v}$ & & 242 & 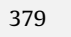 & 7 & 352 & 1120 & 626 & 330 & 944 & 138 & 0 & 21 & 1 & & 2 & & 2 & 4 & 5. & 36 & 4.2 & 2.6 & 362 & 28 & & 6 & 669 & 49 & 8774 & 51.8 \\
\hline $3 \mathrm{~V}$ & $\mathrm{~cm}$ & 256 & 5 & 1 & 268 & 906 & 630 & 343 & 1003 & 139 & & 213 & A & & 2 & & & 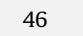 & & 3 & & 7 & 526 & 35 & & & 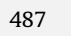 & & 7239 & 26.8 \\
\hline $4 \mathrm{~W}$ & 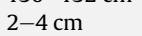 & 197 & 12 & 11. & 238 & 796 & 506 & 287 & 826 & 117 & 39 & 18 & 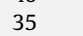 & & 2 & 11 & 1 & 4 & 4.8 & 27 & 3.4 & 2.1 & 796 & 35 & 22 & 309 & 248 & 37 & 5659 & 48.5 \\
\hline $4 \mathrm{~W}$ & $2-4 \mathrm{~cm}$ & 175 & 528 & 10.8 & 238 & 730 & 448 & 260 & 742 & 110 & 571 & 164 & 32 & 185 & 22 & 103 & 16 & 37 & 4.5 & 24 & 3.0 & 1.8 & 737 & 31 & 20 & 307 & 238 & 0.42 & 5681 & 48.9 \\
\hline $4 \mathrm{~W}$ & 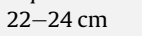 & & & & & 24 & & & 837 & 111 & & 1 & & & 2 & 10 & & 3 & & 2 & & 4.0 & 429 & 28 & & & & & 16 & 46.6 \\
\hline $4 W$ & $\begin{array}{l}22-24 \mathrm{~cm} \\
\text { duplicate }\end{array}$ & 230 & 401 & 9.2 & 202 & 770 & 469 & 270 & 795 & 109 & 573 & 162 & 30 & 175 & 22 & 9 & 16 & 3 & 4.3 & 23 & 3.1 & 3.8 & 438 & 29 & 23 & 402 & 527 & 0.35 & 7904 & 43.4 \\
\hline $4 \mathrm{~V}$ & & & & & & & & & 0 & 1 & & & & & 2 & & & & & & & & & & & & & & 55 & 0 \\
\hline 41 & & & 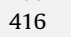 & 9. & & 54 & 560 & 322 & 5 & 124 & 5 & 1 & & & 2 & & & & 5. & & & 2. & 472 & 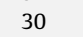 & & 34 & 54 & 29 & 7307 & 42.3 \\
\hline $4 \mathrm{~V}$ & & 1 & 41 & & 225 & 626 & 469 & 286 & 785 & 10 & & 13 & & & 1 & 9 & & 3 & 4. & & & & 369 & 23 & & & 6 & & 15 & 48.9 \\
\hline $4 W$ & $\begin{array}{l}74-76 \mathrm{~cm} \\
\text { duplicate }\end{array}$ & 213 & 437 & 10.0 & 305 & 846 & 482 & 303 & 818 & 110 & 563 & 148 & 27 & 16 & 2 & ( & 1 & 35 & 4.5 & 2 & 3.5 & 3.8 & 396 & 22 & 24 & 377 & 537 & 0.54 & 9675 & 43.5 \\
\hline $4 \mathrm{n}$ & 9 & 33 & & & & & & & 1052 & 14 & & & & & 2 & & & & & & & 4. & 13 & & & & & & 10,465 & 45.9 \\
\hline $4 \mathrm{~V}$ & & 235 & $4 \mathrm{c}$ & & & & & & 899 & 12 & & & & & 2 & & & & & 27 & & 2. & 535 & 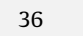 & & & & & & 40.7 \\
\hline $4 \mathrm{~W}$ & $142-144 \mathrm{~cm}$ & 213 & 496 & 12.5 & 243 & 801 & 533 & 323 & 856 & 122 & 611 & 166 & 31 & 184 & 23 & 106 & 17 & 41 & 5.0 & 27 & 3.7 & 2.7 & 453 & 29 & 21 & 348 & 469 & 0.40 & 7429 & 39.7 \\
\hline NOD-A & $\begin{array}{l}\text { leached } \\
\text { USGS std. }\end{array}$ & 385 & 2763 & 6132 & 20,412 & 2100 & 91 & 1570 & 007 & 316 & 1363 & 281 & 6 & 294 & 49 & 290 & 59 & 173 & 27 & 172 & 26 & 1333 & 11,217 & 221 & 26 & 283 & 34 & 15 & 1743 & 0.45 \\
\hline NOD-P-1 & $\begin{array}{l}\text { leached } \\
\text { USGS std. }\end{array}$ & 223 & 1204 & 8679 & 3005 & 2019 & 1071 & 1509 & 4783 & 420 & 1831 & 425 & 93.4 & 390 & 64 & 342 & 62 & 152 & 2 & 167 & 22 & 326 & 2400 & 87 & 25 & 122 & 93 & 1.64 & 2569 & 0.14 \\
\hline AGV & & & & & & ס/ & 2 & 40 & 11 & & 33 & & & & 0.6 & & & & 0. & & 0. & 0.3 & 13 & & & & & & & \\
\hline BCR-2 & & n.a. & M.d. & n. & 13,856 & 347 & 39 & 27 & 56 & 6.6 & 31 & 6.90 & 1.9 & 7.01 & 1.16 & 6.63 & 1.33 & 3.5 & 0.54 & 3.49 & 0.50 & 0.29 & 10 & 6.24 & 1.70 & n.a. & n.a. & n.a. & n.a. & n.a. \\
\hline
\end{tabular}



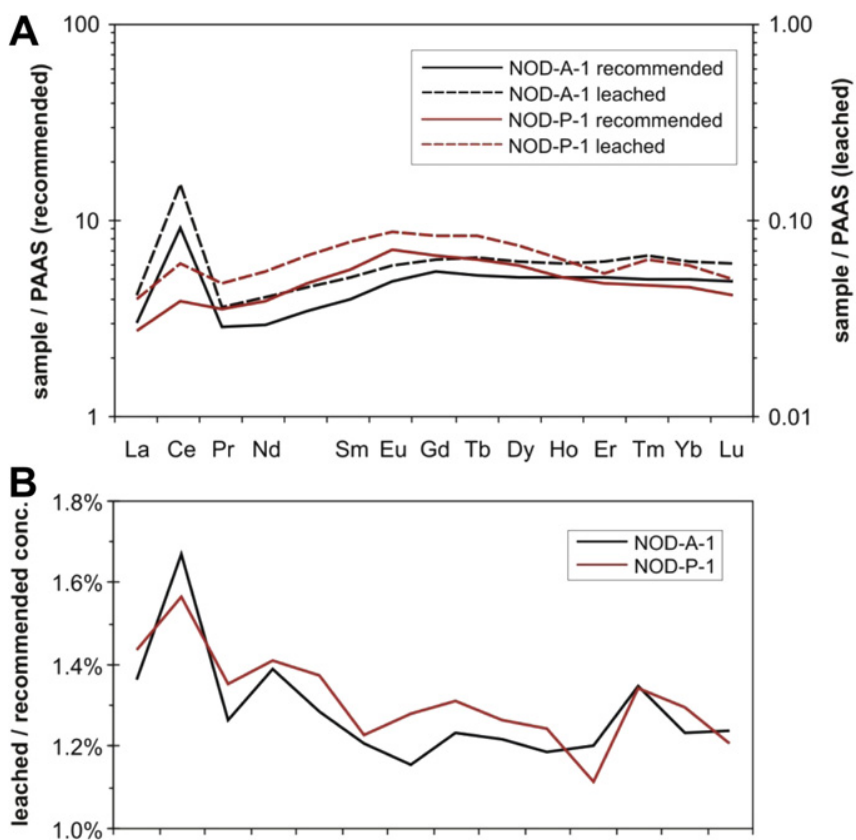

La Ce Pr Nd Sm Eu Gd Tb Dy Ho Er Tm Yb Lu

Fig. 2. Post-Archean Australian Average Shale (PAAS) normalised recommended (Axelsson et al., 2002) and extracted REE concentrations for USGS ferromanganese nodule standard materials NOD-A-1 and NOD-P-1 (see Table 2). Although the extracted relative REE concentrations closely resemble the recommended concentrations (A), only $1.2-1.8 \%$ of the ferromanganese nodule material was extracted during the onehour reductive leach (B). PAAS data are from McLennan (1989).

(i.e., trace metal concentrations per gram of leached sediment) are highly reproducible in both their concentrations (Table 2) and PAAS-normalised REE patterns (Fig. 3b-d).

The $\mathrm{Sr}$ isotope results displayed in Table 1 are higher than present-day seawater $\left({ }^{87} \mathrm{Sr} /{ }^{86} \mathrm{Sr}_{\text {seawater }} \sim 0.70917\right)$ (Henderson et al., 1994) and indicate that the aluminosilicate fraction in the sediment was also partially attacked, a feature that has been observed previously (Gutjahr et al., 2007). Nevertheless the REE patterns shown in Fig. 3 suggest that the dominant REE (and hence $\mathrm{Nd}$ ) signal is derived from the Fe-Mn oxyhydroxide phase, otherwise the PAAS-normalised patterns would be flat and would not display a shale-normalised MREE enrichment indicative of dissolved Fe-Mn oxyhydroxide phases (Haley et al., 2004). Moreover, based on the mass balance calculations presented in Gutjahr et al. (2007) we consider the extracted authigenic $\mathrm{Fe}-\mathrm{Mn}$ oxyhydroxide signal insensitive to detrital contamination. We want to emphasize that Holocene authigenic Nd isotope compositions from nearby cores along the Blake Ridge reproduced the present-day NADW Nd isotope composition below $3200 \mathrm{~m}$ water depth, whereas more shallow locations were biased by downslope transport of particles that acquired their authigenic Nd isotope signal on the shelves (Gutjahr et al., 2008).

\subsection{Neodymium isotope compositions}

The bottom water Nd isotope evolution, as recorded by the Fe-Mn oxyhydroxides at Site 1060 , only shows a small but significant variability between 46 and 35 ka BP (Figs. 4 and 5). For comparison we also show the $\mathrm{Nd}$ isotope evolution across the transition from the Last Glacial Maximum (LGM) to the Holocene recorded in adjacent core KNR140-12JPC. This core, located some $770 \mathrm{~m}$ deeper than site 1060 on the Blake Ridge at $4250 \mathrm{~m}$, contained typical LNADW $\varepsilon_{\mathrm{Nd}}$ only since the Younger Dryas. The MIS 3
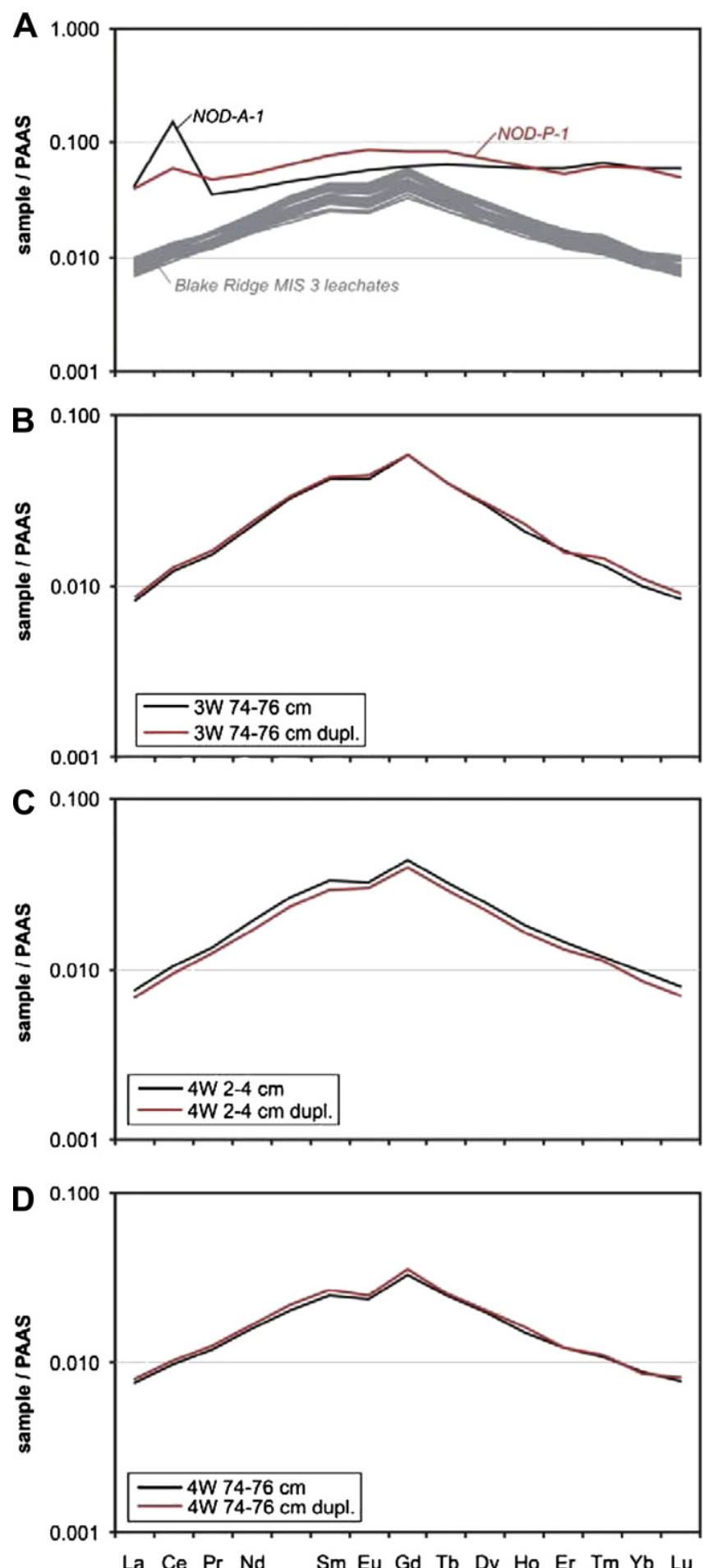

Fig. 3. (A) Post-Archean Australian Average Shale (PAAS) normalised extracted REE concentrations for all leached Blake Ridge MIS 3 sediment samples as well as USGS ferromanganese nodule standard materials NOD-A-1 and NOD-P-1. (B-D) REE multielement plots for individual duplicates that were processed through chemistry separately, displaying a high reproducibility of the extracted chemical compositions. Relative REE abundances are displayed as ng per gram of leached dried powdered sediment prior to normalization to PAAS. PAAS data are from McLennan (1989).

authigenic $\varepsilon_{\mathrm{Nd}}$ signatures were least radiogenic prior to $44 \mathrm{ka} \mathrm{BP}$ $\left(\varepsilon_{\mathrm{Nd}}=-11.2\right)$ and shortly after $39 \mathrm{ka} \mathrm{BP}\left(\varepsilon_{\mathrm{Nd}}=-11.3\right)$, following the termination of $\mathrm{H} 4$ (Fig. 5). Neodymium isotope compositions started to be systematically higher after $44 \mathrm{ka} \mathrm{BP}$ and were most 
radiogenic between 40.7 and $39.1 \mathrm{ka} \mathrm{BP}\left(\varepsilon_{\mathrm{Nd}} \leq-9.8\right)$, which corresponds to the timing of $\mathrm{H} 4$ but the $\mathrm{Nd}$ isotope maximum was longer-lived and its onset clearly predated the onset of H4. Similar to the sortable silt record of the same core (cf. Hoogakker et al., 2007), the Nd isotope evolution does not follow the short-term Dansgaard-Oeschger climate cycles recorded in the Greenland ice cores (Fig. 5). The most radiogenic Nd isotope compositions are, within error, identical to those recorded in core KNR140-12JPC during the LGM and the early deglaciation.

\section{Discussion}

\subsection{NW Atlantic deep water mass evolution during MIS 3}

The main feature apparent in the $\mathrm{Nd}$ isotope record is the absence of 'full strength' (i.e. $\varepsilon_{\mathrm{Nd}}<-13$ ) NADW along the Blake Ridge below $3500 \mathrm{~m}$ water depth at any time between 46 and

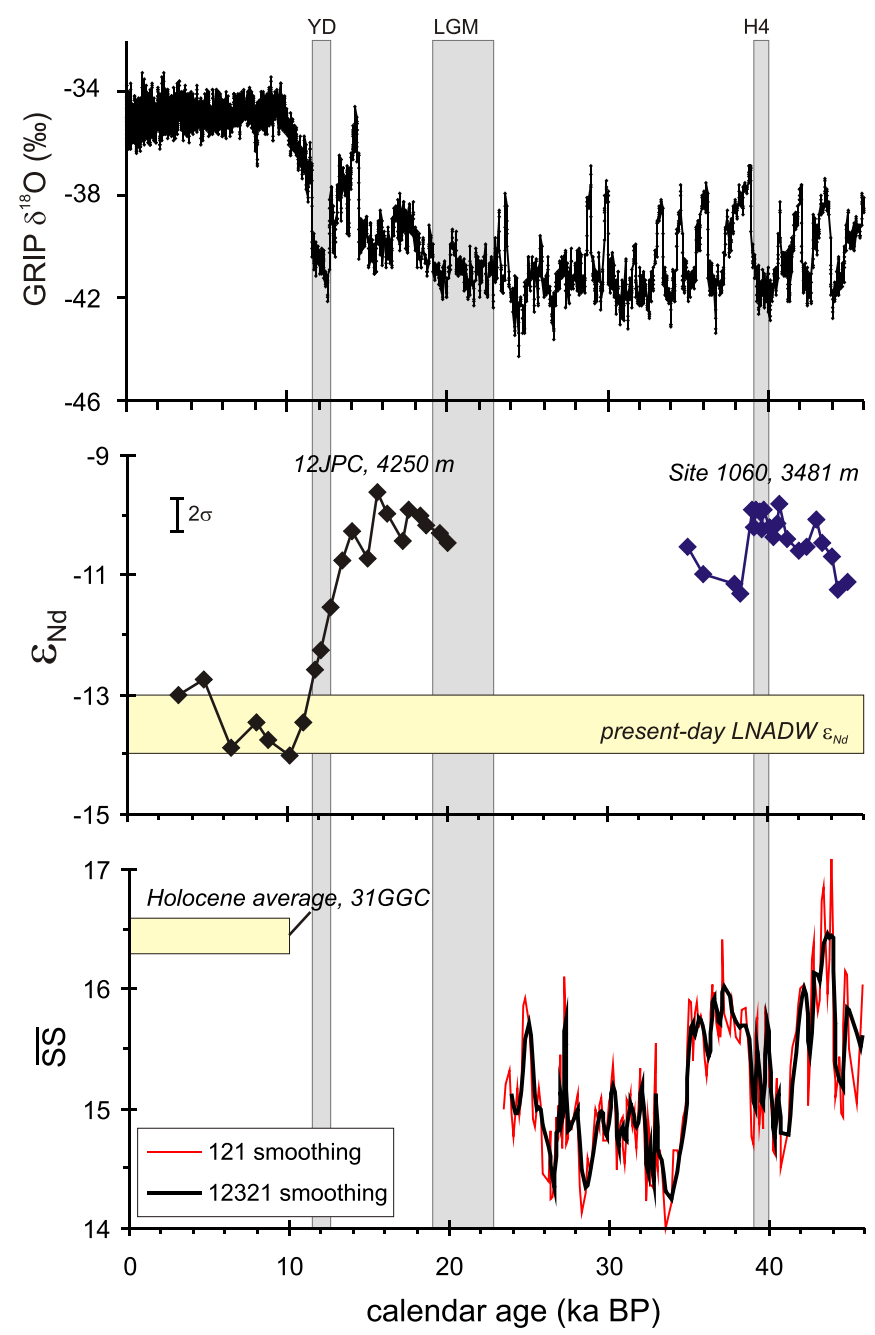

Fig. 4. Authigenic Fe-Mn oxyhydroxide-derived Nd isotope evolution during MIS 3 (45-35 ka BP, this study) and in the transition from the LGM to the Holocene as seen in nearby core KNR140/12JPC (Gutjahr et al., 2008). The Nd isotope record is set in context with the GRIP oxygen isotope record (Grootes et al., 1993; Johnsen et al., 1997) and the sortable silt record from Site 1060 (Hoogakker et al., 2007). The MIS 3 SS data are smoothed following a $(1-2-1)$ and $(1-2-3-2-1)$ weighing scheme in order to better illustrate longer-term grain size trends. Also shown in the lower panel is the average Holocene sortable silt composition for nearby core KNR140/31GGC as presented by Evans and Hall (2008). Timing and duration of $\mathrm{H} 4$ as published in Hemming (2004). Younger Dryas spans the time from 12.9 to $11.7 \mathrm{kyr}$, LGM is highlighted as the interval from 23 to $19 \mathrm{kyr}$.
35 ka BP (Fig. 4). Indeed, much of the record resembles that of the LGM $770 \mathrm{~m}$ deeper. Presence of Lower NADW would have been marked by less radiogenic $\varepsilon_{\mathrm{Nd}}$ values similar to the Holocene (cf. Gutjahr et al., 2008). This assumes that the GNAIW/NADW source water Nd isotope composition during MIS 3 remained as unradiogenic as today $\left(\varepsilon_{\mathrm{Nd}} \sim-13.5\right)$, which is not yet resolved. The best estimate for MIS 3 GNAIW/NADW $\varepsilon_{\text {Nd }}$ is provided by in situ laser ablation Nd isotopic analysis of ferromanganese crust BM1969.05 in $1800 \mathrm{~m}$ water depth on the New England Seamounts (Foster et al., 2007), providing an average GNAIW $\varepsilon_{\mathrm{Nd}}$ during MIS 3 of around -12.5 , which is slightly more radiogenic (by $\sim 1 \varepsilon_{\mathrm{Nd}}$ ) than today. In the interval studied here the least radiogenic $\mathrm{Nd}$ isotopic compositions of -11.1 to -11.3 were recorded at the deeper Blake Ridge before $44 \mathrm{ka} \mathrm{BP}$, immediately preceding $\mathrm{D} / \mathrm{O}$ interstadial 11 and after $39 \mathrm{kaBP}$ during $\mathrm{D} / \mathrm{O}$ interstadial 8. Given the MIS 3 GNAIW $\varepsilon_{\text {Nd }}$ of Foster et al. (2007), these intermediate Nd isotopic compositions suggest some degree of mixing between GNAIW above and SSW bathing the site, assuming a local SSW $\varepsilon_{\mathrm{Nd}}$ composition similar to that at $4250 \mathrm{~m}$ during the LGM-H1 period, i.e. in the range of -9.8 to -10.5 .

Based on high benthic foraminiferal $\delta^{13} \mathrm{C}$ values (epifaunal Cibicidoides wuellerstorfi) indicative of recently ventilated, nutrient depleted water, Vidal et al. (1997) and Elliot et al. (2002) inferred the presence of NADW at water depths above about $3500 \mathrm{~m}$ in the western North Atlantic before and after H4. By analogy, MIS $3 \delta^{13} \mathrm{C}$ records of the infaunal species Uvigerina peregrina from Site 1060 (Vautravers et al., 2004) indicate (somewhat less clearly) the presence of SSW during this time interval rather than GNAIW. Hagen and Keigwin (2002) interpreted epifaunal $\delta^{13} \mathrm{C}$ records to show the interstadial presence of NADW that was replaced by SSW during stadials at neighbouring Site 1059 at $3000 \mathrm{~m}$ water depth on Blake Ridge (see Fig. 1 for core location). The SSW-dominated $\varepsilon_{\mathrm{Nd}}$ during the LGM at nearby Site KNR140/31GGC (3410 m) was around -10.2 (Gutjahr et al., 2008). The $\varepsilon_{\mathrm{Nd}}$ at Site 1060 before Greenland IS 11 and during IS 8 was -11.2 , intermediate between the SSW and the MIS 3 GNAIW end member compositions. Therefore, the slightly less radiogenic Nd isotope composition at Site 1060 during parts of MIS 3 than during the LGM supports the assertion that at least at some stage during MIS 3 GNAIW influence extended deeper than during the LGM, with Site 1060 being located in the transition zone between SSW and GNAIW (see also Vidal et al., 1997; Hoogakker et al., 2007). The Nd isotope record suggests deeper GNAIW ventilation along the Blake Ridge preceding Greenland IS 11 followed by a period of shallowing with SSW dominance, and a resumption of deeper influence of GNAIW at the beginning of Greenland IS 8 (Fig. 5).

The Nd isotopic change towards more radiogenic compositions already initiated at around $44 \mathrm{ka} \mathrm{BP}$ (Fig. 5), several thousand years before the onset of H4. Simultaneously, the mean grain sizes of the sortable silt record at Site 1060 decreased suggesting weaker bottom currents prevailing well before $\mathrm{H} 4$, with smallest mean grain sizes recorded just before H4 (Figs. 4, 5; and Hoogakker et al. (2007)). The slowest flow speeds correspond to the maximum influence of SSW as indicated by the Nd isotopic trends.

Site 1060 was continuously situated either within SSW or in the transition zone between SSW and GNAIW, as deduced from the Nd isotope record. The Greenland ice core $\mathrm{D} / \mathrm{O}$ cycles are resolvable in neither the Nd isotope nor the sortable silt record. The Blake Ridge deep water $\mathrm{Nd}$ isotope and sortable silt records also differ from local reconstructed sea surface temperatures based on the relative abundance of warm and cold surface-dwelling foraminifera (Fig. 5) (Vautravers et al., 2004). The relative abundance of "cold" species suggests generally lowered sea surface temperatures during but also preceding $\mathrm{H} 4$, generally following the Greenland ice core 


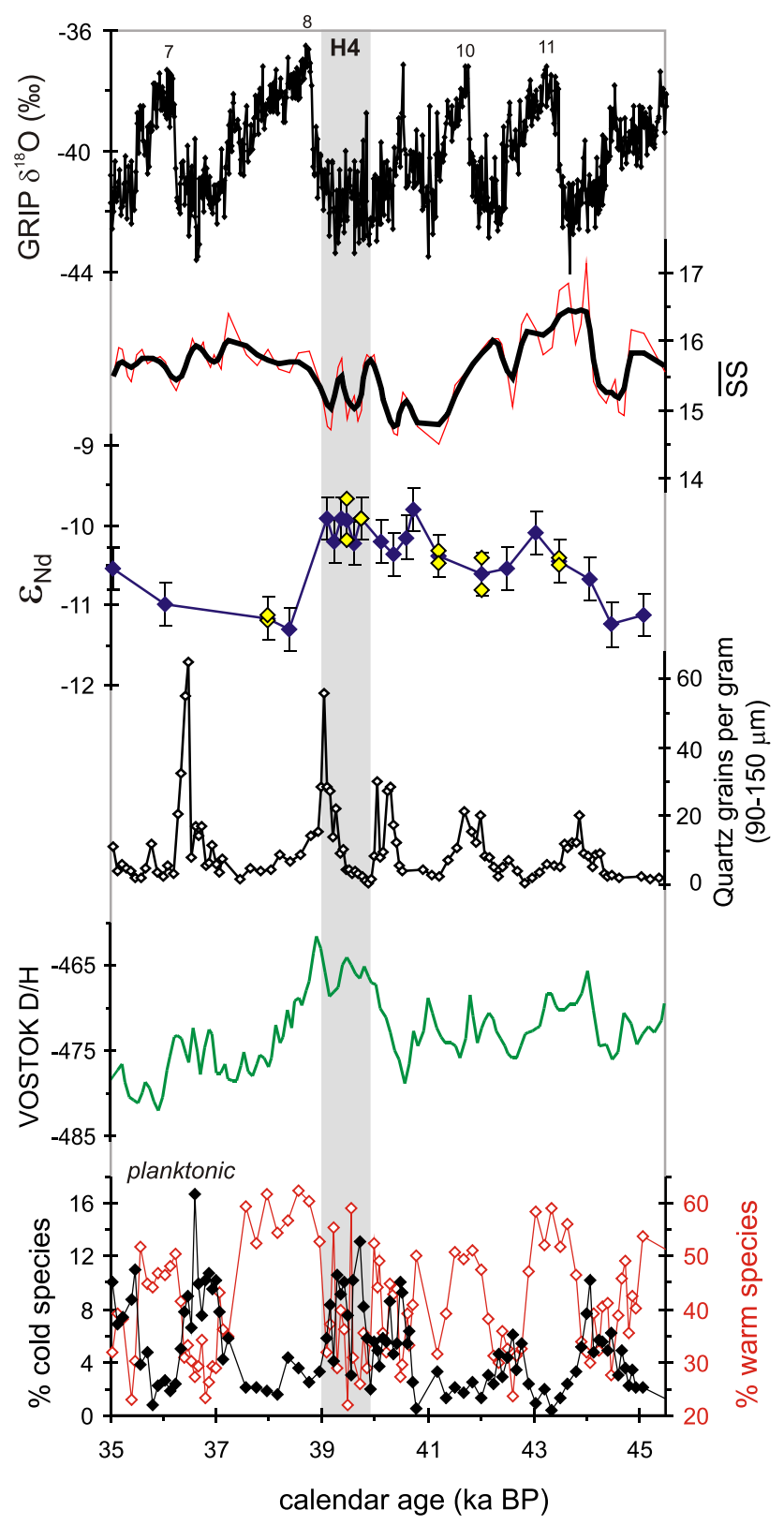

Fig. 5. Authigenic Fe-Mn oxyhydroxide-derived Nd isotope evolution during MIS 3 between 45 and $35 \mathrm{ka} \mathrm{BP}$ (middle panel, blue diamonds), shown alongside the GRIP oxygen isotope record (upper panel) (Grootes et al., 1993; Johnsen et al., 1997), the sortable silt record of Hoogakker et al. (2007), IRD counts from Site 1060 (Vautravers et al., 2004), the Antarctic Vostok D/H record (Jouzel et al., 1987), and the percentages of the surface dwelling warm and cold species planktonic foraminifera from Site 1060 (Vautravers et al., 2004). The color code for the sortable silt record is the same as in Fig. 3. Also shown are individual duplicates of extracted authigenic Fe-Mn oxyhydroxide Nd isotope compositions (yellow diamonds) to illustrate the reproducibility of the chemical extraction procedure (see also Table 1 ). Note that only a smaller $(90-150 \mu \mathrm{m})$ IRD grain size fraction is shown since Site 1060 contains insignificant amounts of IRD in the grain size range $>150 \mu \mathrm{m}$ because of its location outside the main North Atlantic IRD belt. Numbers above the GRIP oxygen isotope record refer to Greenland Interstadials. Oxygen isotope record shown relative to SMOW. Timing of H4 as defined in Hemming (2004).

record (Hagen and Keigwin, 2002; Vautravers et al., 2004). Given that overall the Blake Ridge deep water records do not follow the Greenland climate suggests that the deep water distribution was probably more strongly controlled by changes in the Antarctic, a feature observed also by Shackleton et al. (2000) at $3146 \mathrm{~m}$ water depth on the Iberian margin. Since Site 1060 was always either partially or entirely situated within SSW over the period between 45 and $35 \mathrm{ka}$, this scenario appears likely.

\subsection{Possible Northern controls}

Our data indicate a switch to more SSW-rich bottom waters in the western North Atlantic at the end of Greenland IS12 (in S11), well before $\mathrm{H} 4$ (Stadial 8), and show no fluctuations that might be associated with stadial-interstadial climatic changes between S/IS 11 and 9. Is there any indication of hydrographic shifts or fresh water input at northern latitudes that might have caused the switch? After all, climatic warming/cooling trends in the Northern Hemisphere were important in setting deep water density gradients that should contribute to the observed variability in deep water $\mathrm{Nd}$ isotope compositions in the North Atlantic on glacial-interglacial scales (Clark et al., 2002; Rahmstorf, 2002).

\subsubsection{Shallowing of northern deep waters}

Indicators of vertical variability of the water mass boundary between NSW and SSW have been detected in carbon isotope time series and records of other proxies at various locations in the North Atlantic (Oppo and Lehman, 1995; Zahn et al., 1997; Hagen and Keigwin, 2002; Rasmussen et al., 2003; Peck et al., 2007b), as well as in NW Atlantic Nd isotope records shown here and previously (Gutjahr et al., 2008; Roberts et al., 2010). Based on benthic foraminiferal assemblages, Rasmussen et al. (2003) suggested the presence of relatively warm, poorly ventilated and nutrient rich deep water akin to water of southern origin during Heinrich Events in the southeast Labrador Sea and the presence of NADW in between. In their publication, a reduction of NADW presence prior to Heinrich Events was inferred based on faunal and isotopic changes. Conversely, the appearance of low benthic $\delta^{13} \mathrm{C}$ compositions at deep North Atlantic core sites may potentially reflect not only inflow of Southern Source Water in the deep North Atlantic but sinking of low $\delta^{13} \mathrm{C}$ surface water after brine rejection (Meland et al., 2008; Thornalley et al., 2010).

In a sediment core at $2658 \mathrm{~m}$ water depth from the Gardar Drift, situated on the eastern flank of the Reykjanes Ridge, Oppo and Lehman (1995) observed a large range in benthic $\delta^{13} \mathrm{C}$ values during MIS 3, which were interpreted to reflect changes in NADW production. According to the benthic carbon isotope records, NADW formation was already weak prior to $\mathrm{H} 4$ and did not show additional weakening in response to the massive iceberg discharge. According to Oppo and Lehman (1995), compared with other Greenland stadials Heinrich Events did not stand out as unique periods of decreased NADW production according to their deep water carbon isotope records.

\subsubsection{Northwest European iceberg discharge preceding $H 4$}

Several publications have discussed the importance of iceberg discharge from the Northwest European Ice Sheet (NWEIS) that predated Heinrich Events and potentially preconditioned the North Atlantic for severe THC reductions during HEs (Hall et al., 2006; Peck et al., 2006, 2007a). However, these discharges were hundreds rather than thousands of years before a Heinrich event. While abundant NWEIS-derived IRD contributed to Heinrich precursor events for $\mathrm{H} 1$ and $\mathrm{H} 2$, no significant amount of such IRD was associated with H4 and H5 (Jullien et al., 2006; Scourse et al., 2009). Similarly Snoeckx et al. (1999) labelled H4 as a typical Heinrich event associated with massive discharge from the Laurentide ice sheet with only minor IRD contributions from the NWEIS. Hence it seems unlikely that intensive NW European Ice Sheet disintegration events preceding $\mathrm{H} 4$ were responsible for weakening North Atlantic THC leading to a possible vertical shift in the water mass boundary between GNAIW and SSW at the Blake Ridge. 
In summary, although we find indications for generally reduced Atlantic MOC preceding H4, we cannot identify a northern trigger for the water mass change at lower Blake Ridge more than $3 \mathrm{ka}$ prior to the onset of $\mathrm{H} 4$.

\subsection{North Atlantic-South Atlantic comparison}

From combined benthic carbon isotope and authigenic $\mathrm{Fe}-\mathrm{Mn}$ oxyhydroxide $\mathrm{Nd}$ isotope records in the deep Cape Basin, Piotrowski et al. (2005) argued for variable export of North Atlantic Deep Water into the Southern Ocean on glacial-interglacial timescales as well as during stadial-interstadial stages of MIS 3. Low benthic foraminiferal $\delta^{13} \mathrm{C}$ - indicative of nutrient rich southernsourced deep waters carrying a more radiogenic $\varepsilon_{\mathrm{Nd}}$ - dominated the deep Cape Basin during cold climatic stages, whereas waters with a higher $\delta^{13} \mathrm{C}$ and less radiogenic $\varepsilon_{\mathrm{Nd}}$ characteristic of northern-sourced deep water occupied these core sites during relatively warm stages. Over the course of MIS 3 the apparent NADW contributions to Cape Basin deep water also varied and were strongest during Greenland IS 14, 12 and 8.

In the present North Atlantic study we only recovered the authigenic Fe-Mn oxyhydroxide Nd isotope record for a fraction of MIS 3 (45-35 ka BP), but a direct comparison of the deep Blake Ridge MIS 3 record with the Cape Basin record shows intriguing similarities (Fig. 6). The Nd isotope records in both cores follow a broad trend towards more radiogenic compositions before and during $\mathrm{H} 4$ with the Blake Ridge record apparently slightly leading the Cape Basin record. Clearly the chronologies for both cores have some error but since both core sites are intercalibrated via the GISP2/GRIP age scales of Shackleton et al. (2004) for Greenland

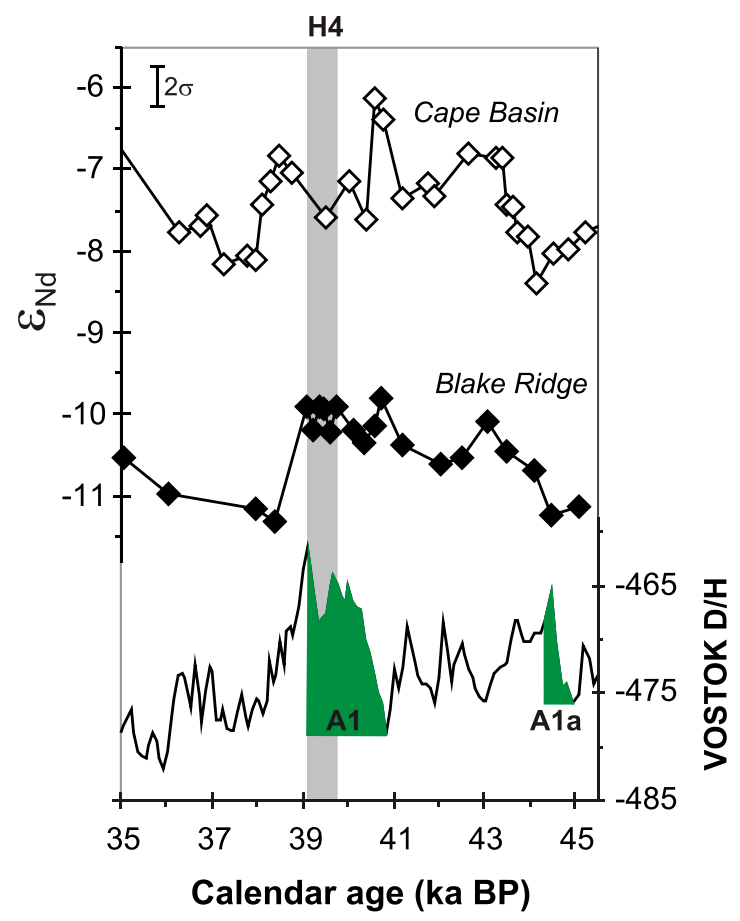

Fig. 6. Comparison of the Blake Ridge and the Cape Basin authigenic Fe-Mn oxyhydroxide Nd isotope record (Piotrowski et al., 2005), shown alongside the VOSTOK D/ $\mathrm{H}$ record (Jouzel et al., 1987) using the revised age scale of Shackleton et al. (2004) for both sites. Antarctic Warming Phases A1 and A1a are highlighted. The Cape Basin Nd isotope record is displayed using the revised chronology for core TNO75-21 of Kissel et al. (2008). To facilitate comparison between the two deep water Nd isotope records, the chronology of Kissel et al. (2008) for Cape Basin core TNO75-21, which is based on the GISP2 chronology of Meese et al. (1997) has been adjusted to the revised GRIP/GISP2 chronology of Shackleton et al. (2004).
Dansgaard-Oeschger cycles this error is most unlikely to be $>1 \mathrm{ka}$. Taken at face value the data suggest that NADW (GNAIW) variability in the western North Atlantic was either contemporaneous or preceded that recorded at the outlet of NADW into the Southern Ocean by a small margin. This is in broad agreement with the magnetically tuned sedimentary records from the North and South Atlantic presented by Kissel et al. (2008), who suggested a propagation time-lag of a North Atlantic signal to the Cape Basin of $860 \pm 220$ years. However, the apparent North Atlantic lead seen here is significantly shorter in the range of $\sim 200-300$ years. If the trends seen at the two core sites are related, they hold important information about inter-basin deep water export.

On the other hand, we also point out that the North Atlantic shift in $\varepsilon_{\mathrm{Nd}}$ at $\sim 44$ ka follows the onset of warmer Antarctic conditions marked by what Hoogakker et al. (2007) termed Antarctic Event $\mathrm{A} 1 \mathrm{a}$, and the return to less radiogenic compositions at 39-38 ka coincides with the end of Antarctic Warm Event A1 (Blunier and Brook, 2001) (Fig. 6). This is consistent with the $\varepsilon_{\mathrm{Nd}}$ signal at Blake Ridge being controlled by events influencing bottom water production and properties around Antarctica. Millennial fluctuations produced a more radiogenic signal (more SSW influence) during warm events that, as noted above, may gain their effect from density changes rather than volume flux. Given the apparent coupling between glacial Antarctic climate and North Atlantic deep water mass distributions it remains to be confirmed whether changes in northwest Atlantic deep water records are capable of leading changes in the deep southeast Atlantic. According to the data presented by Kissel et al. (2008) this should be possible.

\section{Conclusions}

The authigenic $\mathrm{Fe}-\mathrm{Mn}$ oxyhydroxide-derived $\mathrm{Nd}$ isotope record of past deep water from the deeper Blake Ridge in the subtropical western North Atlantic suggests that ODP Site 1060 (3481 m) was most likely located within the mixing zone between northern- and southern-sourced water prior to $44 \mathrm{ka} \mathrm{BP}$ preceding $\mathrm{D} / \mathrm{O}$ interstadial 11 and after $39 \mathrm{ka}$ BP during D/O interstadial 8. Between $44 \mathrm{ka}$ and $39 \mathrm{ka}$ it was located within SSW. Heinrich iceberg discharge event 4 (H4) was marked by Nd isotopic compositions as radiogenic as during the LGM. However, the change towards these modified SSW compositions preceded $\mathrm{H} 4$ by more than $3 \mathrm{ka}$; thus the change in Nd isotope compositions seen at Site 1060 was probably not related to $\mathrm{H} 4$. The Nd isotope record does not follow the Greenland ice core $\mathrm{D} / \mathrm{O}$ cycles, but is consistent with control by Antarctic millennial warming and cooling events. The onset of $\mathrm{D} / \mathrm{O}$ interstadial 8 immediately following $\mathrm{H} 4$ was marked by a $\sim 1.5 \varepsilon_{\mathrm{Nd}}$ jump towards less radiogenic compositions indicative of re-invigorated presence of GNAIW/NADW.

\section{Acknowledgments}

We acknowledge Felix Oberli, Claudine Stirling, Helen Williams, Sarah Woodland, Mark Rehkämper, Heiri Baur, Urs Menet, Donat Niederer, Bruno Rütsche and Andreas Süsli at the ETHZ for their help in keeping the MC-ICPMS running smoothly and their support in the clean labs and with the computers. Jason Day and Mervyn Greaves (University of Cambridge) provided invaluable support with sample preparation and analysis for major and trace element analysis. The manuscript benefited from communications with Alex Piotrowski. This research used samples and/or data provided by the Ocean Drilling Program (ODP). ODP is sponsored by the U.S. National Science Foundation and participating countries (Natural Environment Research Council in UK) under management of Joint Oceanographic Institutions (JOI), Nick McCave and Babette Hoogakker acknowledge financial support from the Isaac Newton 
Trust, Cambridge. Constructive criticism by Alex Piotrowski and one anonymous reviewer improved the quality of the manuscript. Colin Murray-Wallace and Tina van de Flierdt are acknowledged for editorial handling.

\section{References}

Adkins, J.F., McIntyre, K., Schrag, D.P., 2002. The salinity, temperature, and $\delta^{18} \mathrm{O}$ of the glacial deep ocean. Science 298, 1769-1773.

Amos, A.F., Gordon, A.L., Schneide, E.D., 1971. Water masses and circulation patterns in region of Blake-Bahama outer ridge. Deep-Sea Research 18, 145-165.

Axelsson, M.D., Rodushkin, I., Ingri, J., Ohlander, B., 2002. Multielemental analysis of Mn-Fe nodules by ICP-MS: optimisation of analytical method. Analyst 127, 76-82.

Bayon, G., German, C.R., Boella, R.M., Milton, J.A., Taylor, R.N., Nesbitt, R.W., 2002. An improved method for extracting marine sediment fractions and its application to $\mathrm{Sr}$ and $\mathrm{Nd}$ isotopic analysis. Chemical Geology 187, 179-199.

Blunier, T., Brook, E.J., 2001. Timing of millennial-scale climate change in Antarctica and Greenland during the last glacial period. Science 291, 109-112.

Bond, G., Broecker, W., Johnsen, S., McManus, J., Labeyrie, L., Jouzel, J., Bonani, G., 1993. Correlations between climate records from North Atlantic sediments and Greenland ice. Nature 365, 143-147.

Brovkin, V., Ganopolski, A., Archer, D., Rahmstorf, S., 2007. Lowering of glacial atmospheric $\mathrm{CO}_{2}$ in response to changes in oceanic circulation and marine biogeochemistry. Paleoceanography 22 Art. No. PA4202.

Chase, Z., Anderson, R.F., Fleisher, M.Q., Kubik, P.W., 2002. The influence of particle composition and particle flux on scavenging of Th, Pa and Be in the ocean. Earth and Planetary Science Letters 204, 215-229.

Clark, P.U., Pisias, N.G., Stocker, T.F., Weaver, A.J., 2002. The role of the thermohaline circulation in abrupt climate change. Nature 415, 863-869.

Cohen, A.S., Onions, R.K., Siegenthaler, R., Griffin, W.L., 1988. Chronology of the pressure-temperature history recorded by a granulite terrain. Contributions to Mineralogy and Petrology 98, 303-311.

Curry, W.B., Oppo, D.W., 2005. Glacial water mass geometry and the distribution of $\delta^{13} \mathrm{C}$ of $\Sigma \mathrm{CO}_{2}$ in the western Atlantic Ocean. Paleoceanography 20 Art. No.: PA1017.

Dansgaard, W., 1993. Evidence for general instability of past climate from a 250-kyr ice-core record. 364, 218-220.

Elliot, M., Labeyrie, L., Duplessy, J.C., 2002. Changes in North Atlantic deep-water formation associated with the Dansgaard-Oeschger temperature oscillations (60-10 ka). Quaternary Science Reviews 21, 1153-1165.

Evans, H.K., Hall, I.R., 2008. Deepwater circulation on Blake Outer Ridge (western North Atlantic) during the Holocene, Younger Dryas, and Last Glacial Maximum. Geochemistry Geophysics Geosystems 9 Art. No. Q03023.

Foster, G.L., Vance, D., 2006. In situ Nd isotopic analysis of geological materials by laser ablation MC-ICP-MS. Journal of Analytical Atomic Spectrometry 21, 288-296.

Foster, G.L., Vance, D., Prytulak, J., 2007. No change in the neodymium isotope composition of deep water exported from the North Atlantic on glacial-interglacial timescales. Geology 35, 37-40.

Frank, M., 2002. Radiogenic isotopes: tracers of past ocean circulation and erosional input. Reviews of Geophysics 40 Art. No. 1001.

Gherardi, J.-M., Labeyrie, L., Nave, S., Francois, R., McManus, J.F., Cortijo, E., 2009. Glacial-interglacial circulation changes inferred from ${ }^{231} \mathrm{~Pa} /{ }^{230} \mathrm{Th}$ sedimentary record in the North Atlantic region. Paleoceanography 24 Art. No. PA2204.

Goldstein, S.L., Hemming, S.R., 2003. Long-lived isotopic tracers in oceanography, paleoceanography, and ice-sheet dynamics. Treatise on Geochemistry 6, 453-489.

Grootes, P.M., Stuiver, M., White, J.W.C., Johnsen, S., Jouzel, J., 1993. Comparison of oxygen isotope records from the GISP2 and GRIP Greenland ice cores. Nature 366, 552-554.

Gutjahr, M., Frank, M., Stirling, C.H., Klemm, V., van de Flierdt, T., Halliday, A.N., 2007. Reliable extraction of a deepwater trace metal isotope signal from $\mathrm{Fe}-\mathrm{Mn}$ oxyhydroxide coatings of marine sediments. Chemical Geology 242, 351-370.

Gutjahr, M., Frank, M., Stirling, C.H., Keigwin, L.D., Halliday, A.N., 2008. Tracing the Nd isotope evolution of North Atlantic Deep and Intermediate Waters in the western North Atlantic since the Last Glacial Maximum from Blake Ridge sediments. Earth and Planetary Science Letters 266, 61-77.

Hagen, S., Keigwin, L.D., 2002. Sea-surface temperature variability and deep water reorganisation in the subtropical North Atlantic during Isotope Stage 2-4. Marine Geology 189, 145-162.

Haley, B.A., Klinkhammer, G.P., McManus, J., 2004. Rare earth elements in pore waters of marine sediments. Geochimica et Cosmochimica Acta 68, 1265-1279.

Hall, I.R., Moran, S.B., Zahn, R., Knutz, P.C., Shen, C.C., Edwards, R.L., 2006. Accelerated drawdown of meridional overturning in the late-glacial Atlantic triggered by transient pre-H event freshwater perturbation. Geophysical Research Letters 33 Art. No. L16616.

Hemming, S.R., 2004. Heinrich events: massive late pleistocene detritus layers of the North Atlantic and their global climate imprint. Reviews of Geophysics 42 Art. No. RG1005.

Henderson, G.M., Martel, D.J., Onions, R.K., Shackleton, N.J., 1994. Evolution of seawater Sr-87/Sr-86 over the last $400 \mathrm{ka}$ - the absence of glacial-interglacial cycles. Earth and Planetary Science Letters 128, 643-651.
Hoogakker, B.A.A., McCave, I.N., Vautravers, M.J., 2007. Antarctic link to deep flow speed variation during Marine Isotope Stage 3 in the western North Atlantic. Earth and Planetary Science Letters 257, 463-473.

Horwitz, E.P., Chiarizia, R., Dietz, M.L., 1992. A novel strontium-selective extraction chromatographic resin. Solvent Extraction and Ion Exchange 10, 313-336.

Jacobsen, S.B., Wasserburg, G.J., 1980. Sm-Nd isotopic evolution of chondrites. Earth and Planetary Science Letters 50, 139-155.

Jeandel, C., 1993. Concentration and isotopic composition of $\mathrm{Nd}$ in the South Atlantic Ocean. Earth and Planetary Science Letters 117, 581-591.

Jeandel, C., Bishop, J.K., Zindler, A., 1995. Exchange of neodymium and its isotopes between seawater and small and large particles in the Sargasso Sea. Geochimica et Cosmochimica Acta 59, 535-547.

Johannesson, K.H., Burdige, D.J., 2007. Balancing the global oceanic neodymium budget: evaluating the role of groundwater. Earth and Planetary Science Letters 253, 129-142.

Johnsen, S.J., Clausen, H.B., Dansgaard, W., Fuhrer, K., Gundestrup, N., Hammer, C.U. Iversen, P., Jouzel, J., Stauffer, B., Steffensen, J.P., 1992. Irregular glacial interstadials recorded in a new Greenland ice core. 359, 311-313.

Johnsen, S.J., Clausen, H.B., Dansgaard, W., Gundestrup, N.S., Hammer, C.U., Andersen, U., Andersen, K.K., Hvidberg, C.S., DahlJensen, D., Steffensen, J.P. Shoji, H., Sveinbjornsdottir, A.E., White, J., Jouzel, J., Fisher, D., 1997. The $\delta^{18} \mathrm{O}$ record along the Greenland Ice Core Project deep ice core and the problem of possible Eemian climatic instability. Journal of Geophysical Research-Oceans 102, 26397-26410.

Jouzel, J., Lorius, C., Petit, J.R., Genthon, C., Barkov, N.I., Kotlyakov, V.M., Petrov, V.M., 1987. Vostok ice core - a continuous isotope temperature record over the las climatic cycle (160,000 years). Nature 329, 403-408.

Jullien, E., Grousset, F.E., Hemming, S.R., Peck, V.L., Hall, I.R., Jeantet, C., Billy, I., 2006. Contrasting conditions preceding MIS3 and MIS2 Heinrich events. Global and Planetary Change 54, 225-238.

Keigwin, L.D., Boyle, E.A., 2008. Did North Atlantic overturning halt 17,000 years ago? Paleoceanography 23 Art. No. PA1101.

Keigwin, L.D., Curry, W.B., Lehman, S.J., Johnsen, S., 1994. The role of the deep ocean in North Atlantic climate change between 70 and $130 \mathrm{kyr}$ ago. Nature 371, 323-326.

Kissel, C., Laj, C., Piotrowski, A.M., Goldstein, S.L., Hemming, S.R., 2008. Millennialscale propagation of Atlantic deep waters to the glacial Southern Ocean. Paleoceanography 23 Art. No. PA2102.

Klevenz, V., Vance, D., Schmidt, D.N., Mezger, K., 2008. Neodymium isotopes in benthic foraminifera: core-top systematics and a down-core record from the Neogene south Atlantic. Earth and Planetary Science Letters 265, 571-587.

Lacan, F., Jeandel, C., 2004a. Subpolar Mode Water formation traced by neodymium isotopic composition. Geophysical Research Letters 31 Art. No. L14306.

Lacan, F., Jeandel, C., 2004b. Neodymium isotopic composition and rare earth element concentrations in the deep and intermediate Nordic Seas: constraints on the Iceland Scotland Overflow Water signature. Geochemistry Geophysics Geosystems 5 Art. No. Q11006.

Lacan, F., Jeandel, C., 2005a. Neodymium isotopes as a new tool for quantifying exchange fluxes at the continent-ocean interface. Earth and Planetary Science Letters 232, 245-257.

Lacan, F., Jeandel, C., 2005b. Acquisition of the neodymium isotopic composition of the North Atlantic Deep Water. Geochemistry Geophysics Geosystems 6 Art. No. Q12008.

Lippold, J., Grutzner, J., Winter, D., Lahaye, Y., Mangini, A., Christl, M., 2009. Does sedimentary ${ }^{231} \mathrm{~Pa} /{ }^{230} \mathrm{Th}$ from the Bermuda Rise monitor past Atlantic Meridional Overturning Circulation? Geophysical Research Letters 36 Art. No.: L12601.

Lynch-Stieglitz, J., Adkins, J.F., Curry, W.B., Dokken, T., Hall, I.R., Herguera, J.C. Hirschi, J.J.M., Ivanova, E.V., Kissel, C., Marchal, O., Marchitto, T.M., McCave, I.N., McManus, J.F., Mulitza, S., Ninnemann, U., Peeters, F., Yu, E.-F., Zahn, R., 2007. Atlantic meridional overturning circulation during the Last Glacial Maximum. Science 316, 66-69.

McLennan, S.M., 1989. Rare earth elements in sedimentary rocks - influence of provenance and sedimentary processes. Reviews in Mineralogy 21, 169-200.

McManus, J.F., Francois, R., Gherardi, J.M., Keigwin, L.D., Brown-Leger, S., 2004 Collapse and rapid resumption of Atlantic meridional circulation linked to deglacial climate changes. Nature 428, 834-837.

Meese, D.A., Gow, A.J., Alley, R.B., Zielinski, G.A., Grootes, P.M., Ram, M., Taylor, K.C. Mayewski, P.A., Bolzan, J.F., 1997. The Greenland Ice Sheet Project 2 depth-age scale: methods and results. Journal of Geophysical Research-Oceans 102, 26411-26423.

Meland, M.Y., Dokken, T.M., Jansen, E., Hevroy, K., 2008. Water mass properties and exchange between the Nordic seas and the northern North Atlantic during the period 23-6 ka: benthic oxygen isotopic evidence. Paleoceanography 23.

NGRIP Members, 2004. High-resolution record of Northern Hemisphere climate extending into the last interglacial period. Nature 431 (7005), 147-151.

Oppo, D.W., Lehman, S.J., 1995. Suborbital timescale variability of North-Atlantic deep-water during the past 200,000 years. Paleoceanography 10, 901-910.

Palmer, M.R., Elderfield, H., 1985. Variations in the Nd isotopic composition of foraminifera from Atlantic Ocean sediments. Earth and Planetary Science Letters 73, 299-305.

Peck, V.L., Hall, I.R., Zahn, R., Elderfield, H., Grousset, F., Hemming, S.R., Scourse, J.D., 2006. High resolution evidence for linkages between NW European ice sheet instability and Atlantic meridional overturning circulation. Earth and Planetary Science Letters 243, 476-488. 
Peck, V.L., Hall, I.R., Zahn, R., Grousset, F., Hemming, S.R., Scourse, J.D., 2007a. The relationship of Heinrich Events and their European precursors over the past 60 ka BP: a multi-proxy ice-rafted debris provenance study in the North East Atlantic. Quaternary Science Reviews 26, 862-875.

Peck, V.L., Hall, I.R., Zahn, R., Scourse, J.D., 2007b. Progressive reduction in NE Atlantic intermediate water ventilation prior to Heinrich events: response to NW European ice sheet instabilities? Geochemistry Geophysics Geosystems 8 Art. No. Q01N10.

Piepgras, D.J. Wasserburg, G.J. 1987. Rare earth element transport in the western North Atlantic inferred from Nd isotopic observations. Geochimica et Cosmochimica Acta 51, 1257-1271.

Piotrowski, A.M., Goldstein, S.L., Hemming, S.R., Fairbanks, R.G., 2005. Temporal relationships of carbon cycling and ocean circulation at glacial boundaries. Science 307, 1933-1938.

Rahmstorf, S., 2002. Ocean circulation and climate during the past 120,000 years Nature 419, 207-214.

Rasmussen, T.L., Oppo, D.W., Thomsen, E., Lehman, S.J., 2003. Deep sea records from the southeast Labrador Sea: ocean circulation changes and ice-rafting events during the last 160,000 years. Paleoceanography 18 Art. No. 1018.

Rickli, J., Frank, M., Halliday, A.N., 2009. The hafnium-neodymium isotopic composition of Atlantic seawater. Earth and Planetary Science Letters 280, $118-127$.

Roberts, N.L. Piotrowski, A.M., McManus, J.F, Keigwin, L.D, 2010. Synchronous deglacial overturning and water mass source changes. Science 327, 75-78.

Rutberg, R.L., Hemming, S.R., Goldstein, S.L., 2000. Reduced North Atlantic deep water flux to the glacial Southern Ocean inferred from neodymium isotope ratios. Nature 405, 935-938.

Schmitz, W.J., McCartney, M.S., 1993. On the North Atlantic circulation. Reviews of Geophysics 31, 29-49.

Scourse, J.D., Haapaniemi, A.I., Colmenero-Hidalgo, E., Peck, V.L., Hall, I.R., Austin, W. E.N., Knutz, P.C., Zahn, R., 2009. Growth, dynamics and deglaciation of the last British-Irish ice sheet: the deep-sea ice-rafted detritus record. Quaternary Science Reviews 28, 3066-3084.

Shackleton, N.J., Hall, M.A., Vincent, E., 2000. Phase relationships between millennial-scale events 64,000-24,000 years ago. 15, 565-569.

Shackleton, N.J., Fairbanks, R.G., Chiu, T.-C., Parrenin, F., 2004. Absolute calibration of the Greenland time scale: implications for Antarctic time scales and for $\Delta^{14} \mathrm{C}$. Quaternary Science Reviews 23, 1513-1522.
Siddall, M., Khatiwala, S., van de Flierdt, T., Jones, K., Goldstein, S.L., Hemming, S., Anderson, R.F., 2008. Towards explaining the Nd paradox using reversible scavenging in an ocean general circulation model. Earth and Planetary Science Letters 274, 448-461.

Smethie, W.M., Fine, R.A., Putzka, A., Jones, E.P., 2000. Tracing the flow of North Atlantic deep water using chlorofluorocarbons. Journal of Geophysical Research Oceans 105, 14297-14323.

Snoeckx, H., Grousset, F., Revel, M., Boelaert, A., 1999. European contribution of icerafted sand to Heinrich layers H3 and H4. Marine Geology 158, 197-208.

Tachikawa, K., Athias, V., Jeandel, C., 2003. Neodymium budget in the modern ocean and paleo-oceanographic implications. Journal of Geophysical Research Oceans 108 Art. No. 3254

Tachikawa, K., Roy-Barman, M., Michard, A., Thouron, D., Yeghicheyan, D. Jeandel, C., 2004. Neodymium isotopes in the Mediterranean Sea: comparison between seawater and sediment signals. Geochimica et Cosmochimica Acta 68, 3095-3106.

Tanaka, T., Togashi, S., Kamioka, H., Amakawa, H., Kagami, H., Hamamoto, T., Yuhara, M., Orihashi, Y., Yoneda, S., Shimizu, H., Kunimaru, T., Takahashi, K., Yanagi, T., Nakano, T., Fujimaki, H., Shinjo, R., Asahara, Y., Tanimizu, M., Dragusanu, C., 2000. JNdi-1: a neodymium isotopic reference in consistency with La Jolla neodymium. Chemical Geology 168, 279-281.

Thornalley, D.J., Elderfield, H., McCave, I.N. Intermediate and deep water paleoceanography of the northern North Atlantic over the past 21,000 years. Paleocanography 25, Art. No. PA1211.

Vautravers, M.J., Shackleton, N.J., Lopez-Martinez, C., Grimalt, J.O., 2004. Gulf Stream variability during marine isotope stage 3. Paleoceanography 19 Art. No. PA2011.

Vidal, L., Labeyrie, L., Cortijo, E., Arnold, M., Duplessy, J.C., Michel, E., Becque, S., vanWeering, T.C.E., 1997. Evidence for changes in the North Atlantic deep water linked to meltwater surges during the Heinrich events. Earth and Planetary Science Letters 146, 13-27.

Yu, J., Elderfield, H., Piotrowski, A.M., 2008. Seawater carbonate ion- $\delta^{13} \mathrm{C}$ systematics and application to glacial-interglacial North Atlantic ocean circulation. Earth and Planetary Science Letters 271, 209-220.

Zahn, R., Schonfeld, J., Kudrass, H.R., Park, M.H., Erlenkeuser, H., Grootes, P., 1997. Thermohaline instability in the North Atlantic during meltwater events: stable isotope and ice-rafted detritus records from core SO75-26KL, Portuguese margin. Paleoceanography 12, 696-710. 